Review article

\title{
Lipid nanocarriers loaded with natural compounds: Potential new therapies for age related neurodegenerative diseases?
}

\author{
Telma Bezerra Soares ${ }^{\mathrm{a}, \mathrm{b}, 1}$, Luís Loureiro ${ }^{\mathrm{a}, 1}$, Ana Carvalho ${ }^{\mathrm{a}}$, Maria Elisabete C.D. Real Oliveira ${ }^{\mathrm{a}}$, \\ Alberto Dias ${ }^{\mathrm{b}}$, Bruno Sarmento ${ }^{\mathrm{c}, \mathrm{d}, *}$, Marlene Lúcio ${ }^{\mathrm{a}, * *}$ \\ ${ }^{\text {a } C F-U M-U P}$ (Centre of Physics of University of Minho and Porto), Department of Physics, University of Minho, Campus of Gualtar, $4710-057$ Braga, Portugal \\ ${ }^{\mathrm{b}}$ Centre for the research and Technology of Agro-Environmental and Biological Sciences, CITAB-UM, Department of Biology, University of Minho, Campus de Gualtar, \\ 4710-057 Braga, Portugal \\ ${ }^{c}$ i3S - Instituto de Investigação e Inovação em Saúde and INEB - Instituto de Engenharia Biomédica, University of Porto, Rua Alfredo Allen, 208, $4200-135$ Porto, Portugal \\ d CESPU, Instituto de Investigação e Formação Avançada em Ciências e Tecnologias da Saúde, Rua Central de Gandra, 1317, 4585-116 Gandra, Portugal
}

\section{A R T I C L E I N F O}

\section{Keywords:}

Lipid nanocarriers

Natural compounds

Blood brain barrier

Neurooxidation

Neuroinflammation

Targeting

Triggering

\begin{abstract}
A B S T R A C T
Age related neurodegenerative disorders (ARND) are presented as the most debilitating and challenging diseases associated with the central nervous system. Despite the advent of active molecules with a positive role on neurodegenerative mechanisms, many of the current therapeutic strategies remain ineffective in treating or preventing ARND. Lipid nanocarriers have emerged as efficient delivery systems with the capability to cross biological barriers, especially the blood brain barrier (BBB). Also, when associated to natural compounds, lipid nanocarriers have demonstrated to be an interesting alternative to ARND therapies with multiple beneficial effects. This comprehensive review focus on state-of-the-art lipid based nanocarriers for the delivery of natural compounds targeting neurodegeneration. A critical analysis of published reports will be also provided giving indications to researchers about the most promising ARND nanotherapy strategies.
\end{abstract}

\section{Introduction}

Over the last decades, the incidence of diseases associated with central nervous system (CNS) has greatly increased due to demographic changes of population and growth of average life expectancy (Silva et al., 2007). Within these diseases, age related neurodegenerative disorders (ARND) are presented as the most debilitating and challenging characterized by a gradual decline of neurological functions as aging progresses. Of relevance, memory, cognition, motor coordination and even emotional responses are disturbed (Lajtha et al., 2009). Nowadays, ARND are the fourth leading cause of death in the industrialized world and affect, approximately, 10 million people each year (Olesen et al., 2012). According to data gathered in 2010, the
European annual cost associated with the treatment of brain disorders was $€ 798$ billion (Olesen et al., 2012) and in United States was US $\$ 2.5$ trillion and it is expected that these costs will duplicate by 2030 (Trautmann et al., 2016) which emphasizes the need to develop new therapies. Due to complex molecular pathology, most of the current therapeutic strategies remain ineffective for the treatment of ARND as these are focused on a single molecular target and/or focused on treating symptoms without halting neuronal cell loss. To date, there is no curative or preventive therapy for ARND and the few drugs approved by FDA are focused in symptomatic relief (Modi et al., 2009). Other therapeutic obstacle is the fact that bioactive neuroprotective molecules present: small permeability and passage through biological barriers and through the blood brain barrier (BBB); short half-life in the

\footnotetext{
Abbreviations: Ach, acetylcholine; AChE, cholinesterase; AD, Alzheimer disease; AMT, adsorptive mediated transcytosis; APP, amyloid precursor protein; ARND, age related neuro-

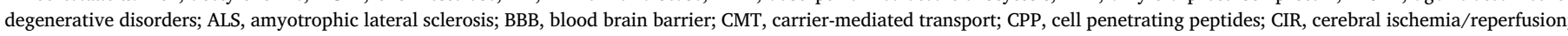

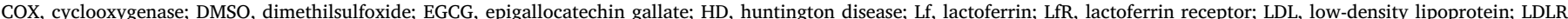

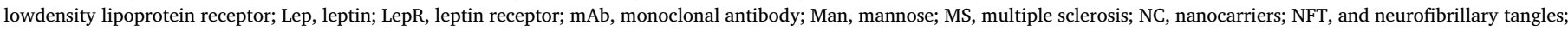

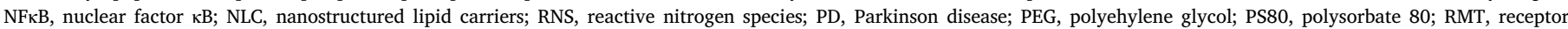

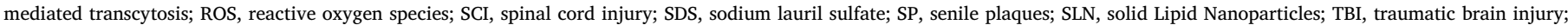
TNF- $\alpha$, tumor necrosis factor $\alpha$; Tf, transferrin; TfR, transferrin receptor

* Corresponding author.

** Corresponding author at: CF-UM -UP(Centre of Physics of University of Minho and Porto), Department of Physics, University of Minho, Escola de Ciências, Campus de Gualtar, 4710057 Braga, Portugal.

E-mail addresses: bruno.sarmento@ineb.up.pt (B. Sarmento), mlucio@fisica.uminho.pt (M. Lúcio).

${ }^{1}$ These authors contributed equally to this work.
} 
systemic circulation; and low bioavailability (Ballabh et al., 2004; Pardridge, 2003; Spuch et al., 2012).

The success of new therapies will be related to their specificity, but also to their multifunctional activities. In light of the overlapping pathways of ARND, one suggested solution is to base effective treatments on agents that have several synergistic effects, especially over a duration that is related with the majority of neurological disorders (Silva, 2006).

For all the mentioned reasons, classical therapies are not effective, and discovering new solutions to deliver bioactives is particularly important in the case of ARND. Accordingly, the EU Joint Programme on Neurodegenerative Disease Research stresses the need to develop novel systems for delivery and targeting of bioactives to the brain (EUJPND, 2012).

Despite the fact that the nanotechnologies are still at infancy stages in clinical neuroscience application, there is a great interest in the potential use of these novel therapeutic solutions (Spuch et al., 2012). Among the various nanoscale delivery systems, lipid nanocarriers have come out as central to ensuring that the delivery process is efficient. These nanocarriers (NC) provide a vehicle that is protected, which shields the bioactives from destruction in an early stage, and also improves their pharmacokinetic profile (Nunes et al., 2015). Having in mind the promising utility of lipid NC in the treatment of ARND and owing to the fact that natural compounds have emerged as a promising hope in the field of drug discovery against these diseases (Devi, 2014), the possibilities emerging from utilizing these strategies to treat CNS disorders are exceptional. Hence, this review discusses and provides a general summary of state-of-the-art of lipid based NC with conveyed natural compounds in a therapeutic perspective targeting neurodegeneration. Additionally, it is presented a critical analysis of published reports, discussing their effectiveness, dissimilarities and developments, as well as, their limitations and achievements giving indications to predict how the lipid NC for natural compounds delivery will evolve and provide better therapeutic solutions for ARND.

\section{Etiology of age related neurodegenerative diseases: brief overview}

ARND are a diverse group of either arbitrary or hereditary disorders that are characterized by a dysfunction of the nervous system that is progressive (Lajtha et al., 2009; Zlokovic, 2008). ARND are multifactorial diseases that arise from slow and irreversible dysfunctions in specific brain areas of the CNS which will, ultimately, delineate the clinical consequences of these conditions. As previously mentioned, aging is a major risk factor to develop neuropathologies although genetics, environmental factors and endogenous dynamics also influence its outset and progression (Lajtha et al., 2009; Zlokovic, 2008). However, the etiological and basic pathological mechanisms that explain and cause these diseases are not yet fully understood (Jain, 2006). The most incident conditions among ARND at a global scale are Alzheimer disease (AD), Huntington disease (HD), spinal cord injury (SCI) amyotrophic lateral sclerosis (ALS), cerebral ischemia/reperfusion (CIR) traumatic brain injury (TBI), Parkinson disease (PD), and multiple sclerosis (MS), (Giacoppo et al., 2015). Although these neurodegenerative conditions exhibit some differences, particularly concerning their etiological pathways and clinical manifestations, these diseases exhibit overlapping features, such as: (i) an imbalanced proteostasis (protein misfolding, aggregation and deposition), (ii) neuroinflammation and (iii) oxidative stress (Spuch et al., 2012). These common features suggest the existence of converging pathways (Fig. 1) in a varying extent of ARND (Durrenberger et al., 2015).

\subsection{The amyloid $\beta$-peptide (A $\beta$ peptide) deposition}

The amyloid $\beta$-peptide ( $\mathrm{A} \beta$ peptide) deposition in the hippocampal and cerebral cortical regions is an etiological factor implicated on the onset of several ARND, namely, AD. This deposition is associated with the senile plaques (SP) and neurofibrillary tangles (NFT) presence. SP are neurotoxic extracellular $A \beta$ peptide deposits build up between nerve cells and derived from the amyloid precursor protein's (APP) secretase cleavage. At synapses, the little clumps of SP may bar sending signals cell-to-cell. They can as well activate the cells of the immune system, which can spark off inflammation and eliminate the cells that are disabled (Tuppo and Arias, 2005). NFT are formed mainly by intracellular aggregates of hyper phosphorylated microtubular tau proteins, whereas SP are extracellular lesions that are complex where the core containing $A \beta$ is encircled by dystrophic neuritis, reactive microglia, interleukins and fibrillary astrocytes. (Richardson et al., 2002). NFT destroy a vital cell transport system based on tau proteins. In AD, tau collapses into twisted strands called tangles in a way such that nutrients together with other supplies that are essential are unable to move through the cells, leading to the cells death. Recent evidences point also to the presence of intermediate aggregation products (A $\beta$ oligomers), in the extracellular or intracellular environment, that can interact with lipid membranes promoting nonspecific ion leakage or may form ion channel-like structures by transformation into annular protofibrils (Sciacca et al., 2012). Eventually, these anomalous channels may compromise neuronal ionic homeostasis (Benilova et al., 2012; Haass and Selkoe, 2007).

\subsection{Oxidative stress}

Oxidative stress is considered to be a key factor in the onset and development of neurodegenerative diseases (Fischer and Maier, 2015). Oxidative stress results from an unbalance between free radicals' production and scavenging. In healthy people, free radicals formation is a usual physiological event critical for the function of CNS. The free radicals are made from metabolism by-products and include peroxynitrite $\left(\mathrm{ONOO}^{-}\right)$, hydrogen peroxide $\left(\mathrm{H}_{2} \mathrm{O}_{2}\right)$, superoxide anion $\left(\cdot \mathrm{O}_{2}{ }^{-}\right)$, hydroxyl radical (HO·), nitroxyl radical $\left(\mathrm{N}_{2} \mathrm{O}_{2}\right)$ and nitric oxide (NO$\left.\cdot\right)$. These free radicals combined are called the reactive oxygen species (ROS) or reactive nitrogen species (RNS) (Chiueh et al., 2000). In a pathological condition, there is an excess of ROS and RNS which damages cellular lipids, proteins, and DNA, thereby, impairing their normal function (Albarracin et al., 2012). The biochemical pathways involved in oxidative stress are complicated, and this complications give numerous therapeutic targets (Chen et al., 2012). Oxidative stress has been showed to factor in several neurodegenerative diseases progression, like AD or PD. Toxicity of ROS and RNS also contributes to: protein misfolding; subsequent cellular apoptosis; mitochondrial dysfunction; and glia cell activation (Fulda et al., 2010). Moreover, A $\beta$ peptide deposition might increase reactive oxygen species (ROS), which are responsible for glutathione depletion and cell vulnerability to oxidant attack (Pinnen et al., 2011).

\subsection{Neuroinflammation}

Neuroinflammation is especially relevant in chronic ARND such as $\mathrm{PD}$ and $\mathrm{AD}$ and it is still looked at as a downstream consequence in regards to the amyloid hypothesis, in which $A \beta$ peptide accumulation in the CNS induces microglia activation, triggering a pro-inflammatory cascade, whose consequences are the release of substances that are potentially neurotoxic that include cytokines, ROS, RNS, chemokines, and different proteolytic enzymes, resulting in neurodegeneration (Eikelenboom et al., 2006; Griffin, 2006). It had been proposed that the microglia and astrocytes activation by upregulation of complement proteins, cytokines, and cyclooxygenase (COX), can result in tau phosphorylation and neurofibrillary tangles formation (NFT) (Zotova et al., 2010). This provides an environment that is neuroinflammatory and is believed to encourage neurodegeneration (Fischer and Maier, 2015). When astrocytes are activated for an extended period of time, they can induce the release of extracellular matrix proteins, which can 


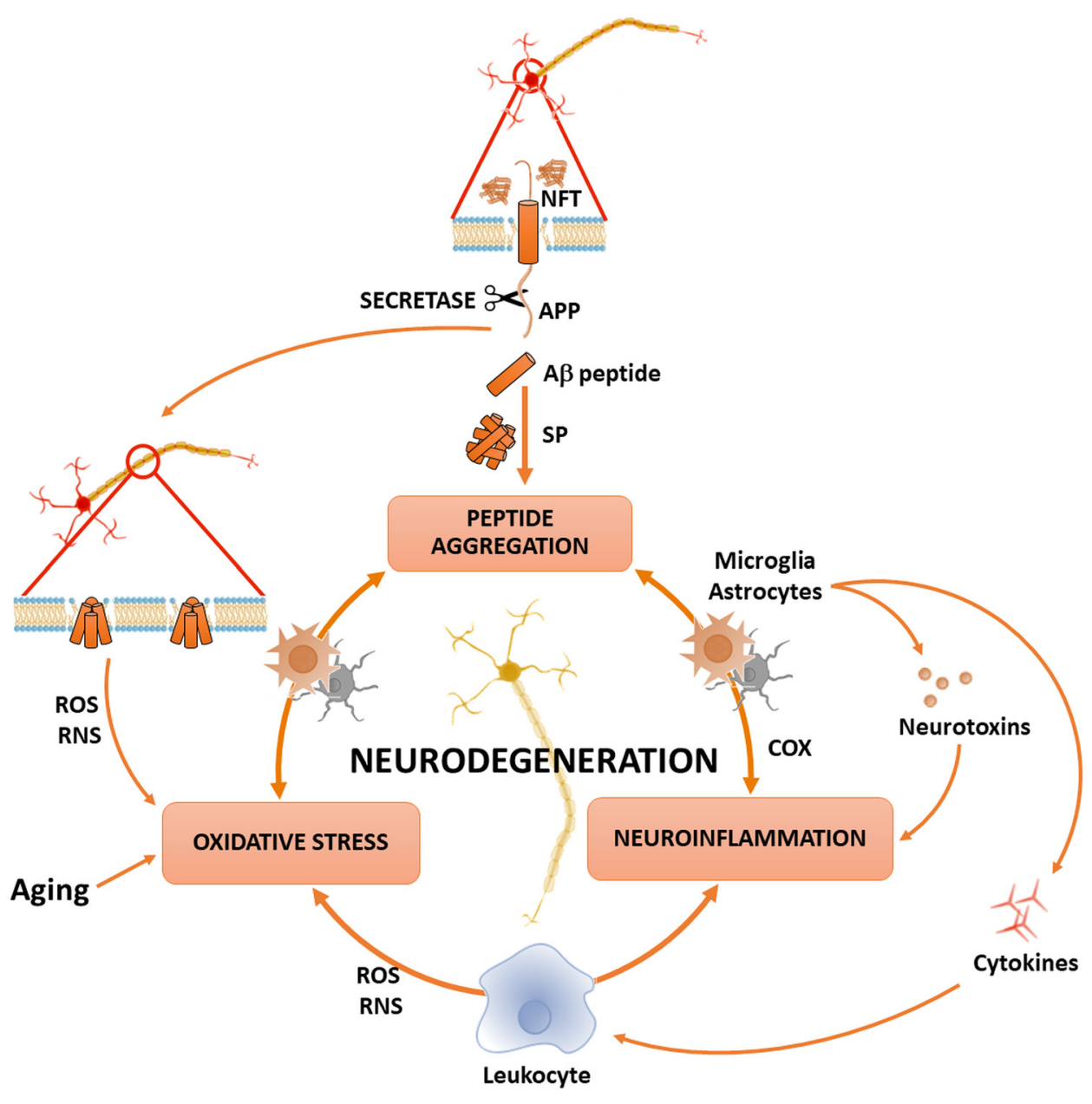

Fig. 1. Pathways involved in neurodegeneration.

Secretase promotes the cleavage of amyloid precursor protein (APP) which is determinant in Amyloid $\beta$ (A $\beta$ ) peptide aggregation in senile plaques (SP) and neurofibrillary tangles (NFT). A $\beta$ oligomers may also interact with membranes forming abnormal ion channel-like structures that compromise ionic homeostasis of affected neurons. Mechanisms involved in A $\beta$-mediated neuronal death may include direct neurotoxicity, neuroinflammation (due to cytokine release, complement activation, COX activity) and oxidative stress (by generation of free reactive oxygen and nitrogen species (ROS and NOS)). Activated microglia and astrocytes may release neurotoxins, toxic inflammatory mediators, and free radicals, inducing also neuroinflammation and oxidative stress that ultimately will be responsible by neurodegeneration. cause glial scar formation. This, on one hand, prevents the damaged area from spreading, but limits tissue regeneration on the other hand (Sofroniew, 2009).

\section{Natural compounds as therapeutic alternatives to age related neurodegenerative diseases}

The multifactorial etiology of ARND suggests that drugs and other active compounds should be able to target multiple receptors or ligands so that the therapy can be efficient (Devi, 2014; Ramassamy, 2006; Silva, 2006; Zhang and Tang, 2006). However, the existing classical drug therapies focus on single targets and altering the progression of the disease and alleviating its symptoms without treating the neuronal cell loss (Youdim and Buccafusco, 2005). Since a deficit of acetylcholine (ACh) is well known to exist in AD brain, cholinesterase (AChE) inhibitors are the current drugs that are approved to treatment AD. However, these drugs cannot stop the neurodegeneration process but are only able to aid in memory improvement in the cases of mild dementia. Because the patient's response to AChE inhibitors is dependent on the presynaptic neurons integrity, these agents' therapeutic efficiency decreases with $\mathrm{AD}$ severity. In addition, the side effects of gastrointestinal are associated with their use (Sonkusare et al., 2005). Besides AChE, $N$-methyl-D-aspartate receptors (NMDA) are the new targets of the $\mathrm{AD}$ approved commercial drugs. NMDA are glutamate-gated cation channels with high permeability for calcium $\left(\mathrm{Ca}^{2+}\right)$ that are essential in: CNS development; rhythms generation for locomotion and breathing; and fundamental in learning, neuroplasticity, and memory processes. Although the NMDA receptor physiological activity is critical for ordinary neuronal function, too much of the glutamate receptors activation of NMDA-types causes a $\mathrm{Ca}^{2+}$ excessive influx via the ion channel associated to the receptor, and this excitotoxicity is among the central elements leading to neuronal injury and even resulting in death in both chronic and acute neurologic disorders (Sonkusare et al., 2005). NMDA-receptor antagonists are drugs used in classical AD and other neurodegenerative diseases therapy since they block glutamate excitotoxicity, however these drugs focus in one therapeutic target and it is not completely known whether treatment benefits continue after the progression to moderate-to-severe diseases (Sonkusare et al., 2005). Therefore, the quest for new therapeutic solutions for the treatment of ARND continues and attention must be paid to drugs or other bioactives that are nontoxic and possess several synergic effects. In this context, natural bioactive compounds are emerging as prophylactic and therapeutic agents that execute neuroprotective effects through a variety of actions (Neves et al., 2012; Oliveira et al., 2016; Silva et al., 2004, 2008a; Silva et al., 2008b; Silva et al., 2007): (i) antioxidant action by inactivation of ROS or RNS; regulation of anti-oxidant enzymes (e.g. glutathione, superoxide dismutase and catalase) or protection of mitochondrial function (mitoprotectors); (ii) anti-inflammatory action by reduction the expression of pro-inflammatory factors (for example, the tumor necrosis factor $\alpha$ (TNF- $\alpha$ ) and IL-1 $\beta$ ) that occur in neuroinflammation and by inhibition of nuclear factor $\kappa B(\mathrm{NF \kappa B})$; (iii) $\mathrm{A}_{\beta}$ peptide anti-aggregation and anti-fibrillation effects by providing a boost to the immunological cells' phagocytic properties to assist clear $\mathrm{A} \beta$ senile plaques in $\mathrm{AD}$, and Lewy bodies in $\mathrm{PD}$ or suppress neurotransmitter cleaving enzymes to increase neurotransmitter concentrations in the neurons vicinity or even decrease tau abnormal phosphorylation; and (iv) general neuroprotective actions by having an effect on the neurons' differentiation characteristics. 
Table 1

Natural compounds and their beneficial effects in neurodegenerative condition.

\begin{tabular}{|c|c|c|c|c|}
\hline Compound & Chemical Family & Natural Source & Beneficial Effects & Ref. \\
\hline Baicalin and Baicalein & Flavone & Scutellaria baicalensis & $\begin{array}{l}\text { - Protects against neuronal cell damage induced by } \\
\text { A } \beta \text { accumulation, oxidative stress, and glutamate } \\
\text { induced toxicity } \\
\text { - Promising agent for prevention or therapy of CIR } \\
\text { injury, traumatic brain injury, AD, PD and } \\
\text { dementia }\end{array}$ & $\begin{array}{l}\text { Mu et al. (2011) and Tu et al } \\
\text { (2009) }\end{array}$ \\
\hline Biapigenin & Flavone & Hypericum perforatum & $\begin{array}{l}\text { - Neuroprotective effects against } \mathrm{A} \beta \text { neurotoxicity } \\
\text { and excitotoxic insults } \\
\text { - Mitoprotective }\end{array}$ & $\begin{array}{l}\text { Silva et al. (2004), Silva et al } \\
\text { (2008a,b), Silva et al. (2009) } \\
\text { and Oliveira et al. (2016) }\end{array}$ \\
\hline
\end{tabular}

Chrysin<smiles>COc1cc(-c2cc(=O)c3c(Cl)cc(Cl)cc3o2)cc(OC)c1C</smiles>

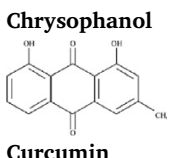

Curcumin<smiles>O=C(/C=C/c1ccc(O)c(O)c1)CC(=O)/C=C/c1ccc(O)c(O)c1</smiles>

Epigallocatechin gallate (EGCG)

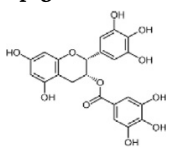

Gallic acid

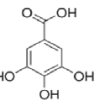

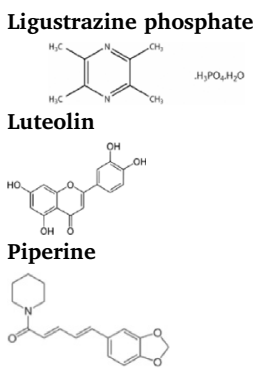

Quercetin<smiles>CC1C(=O)c2c(O)cc(O)cc2OC1c1ccc(O)c(O)c1</smiles>

\section{Resveratrol}

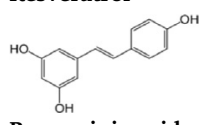

Rosmarinic acid

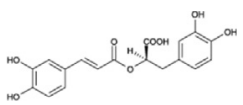

Anthraquinone

Diarylheptanoid (curcuminoid)

Phenolic acid

Alkaloid

Flavone

Alkaloid

Flavonol

Stilbene

Phenolic Acid derivative
Rheum palmatum

Oroxylum indicum, Passiflora caerulea Passiflora incarnata

Curcuma genus

Camellia sinensis

Abundantly present in fruits, tea and nuts

Ligusticum chuanxiong Hort

Salvia tomentosa

Piperis nigri

Different natural sources: Fruits, red wine and Tea

Vitis vinifera and different natural sources

Species of Boraginaceae and subfamily Nepetoideae of Lamiaceae
- Exhibits anti-inflammatory activities and suppresses immuno-inflammatory responses.

- Mitoprotective

- Neuroprotective via a reduction of nitric oxide, TNF- $\alpha$, IL-1 $\beta$, IL- 6 and inhibition of NFKB and COX-2

- Attenuates oxidative damages and cognitive dysfunction associated with $\mathrm{A} \beta$

- Neuroprotective effects against CIR injury

- Inhibition of oxidative stress or neuronal apoptosis-related pathways

- Inhibition of NFKB

- Decreases A $\beta-R O S$ related inflammation

- Inhibits $A \beta$ induced cell death

- Protects neurons from glutamate induced toxicity

- Prevents neuronal loss promoting adult neurogenesis

- Preserves pre and post synaptic proteins.

- Mitoprotective

Walker et al. (2015)

- Inhibits $A \beta$ fibrillation and redirects $A \beta$ aggregation into unstructured, off-pathway oligomers

- Neuroprotective effect against cerebral ischemiainduced oxidative stress

- Neuroprotective against hypoxia/ reoxygenationinduced mitochondrial dysfunctions

- Ameliorated cognitive deficits and cerebral oxidative stress and exerts beneficial effects on behavioral impairments after brain CIR

- Treatment of CIR injury

- Anti-apoptotic and anti-inflammatory activity

- Lowered the intracellular ROS level and increased the neuron survival

- Prevents A $\beta$-induced apoptotic neuronal death

- Anti-inflammatory, anti-depressant, antioxidant and cytoprotective effects.

- Acts on oxidative cascade, cholinergic system and decreases the $\mathrm{A} \beta$ aggregates and NFT in brain tissues

- Increases the neuronal resistance against excitotoxicity by modulation of cell death mechanisms

Zeinali et al. (2017)

Lin et al. (2015)

Ray and Lahiri (2009)

Daglia et al. (2014)

- Free radical quenching, iron chelating and antiinflammatory properties

- Strongly inhibits A $\beta$ fibrillation

- Antioxidant effects

- Neuroprotective effects against $\mathrm{A} \beta$ neurotoxicity and reduced $A \beta$ triggered neuroinflammation

- Potent antioxidant activity associated to its radical scavenging activity

- Anti-inflammatory activity

- Strong anti-A $\beta$ aggregation effects 
Table 1 (continued)

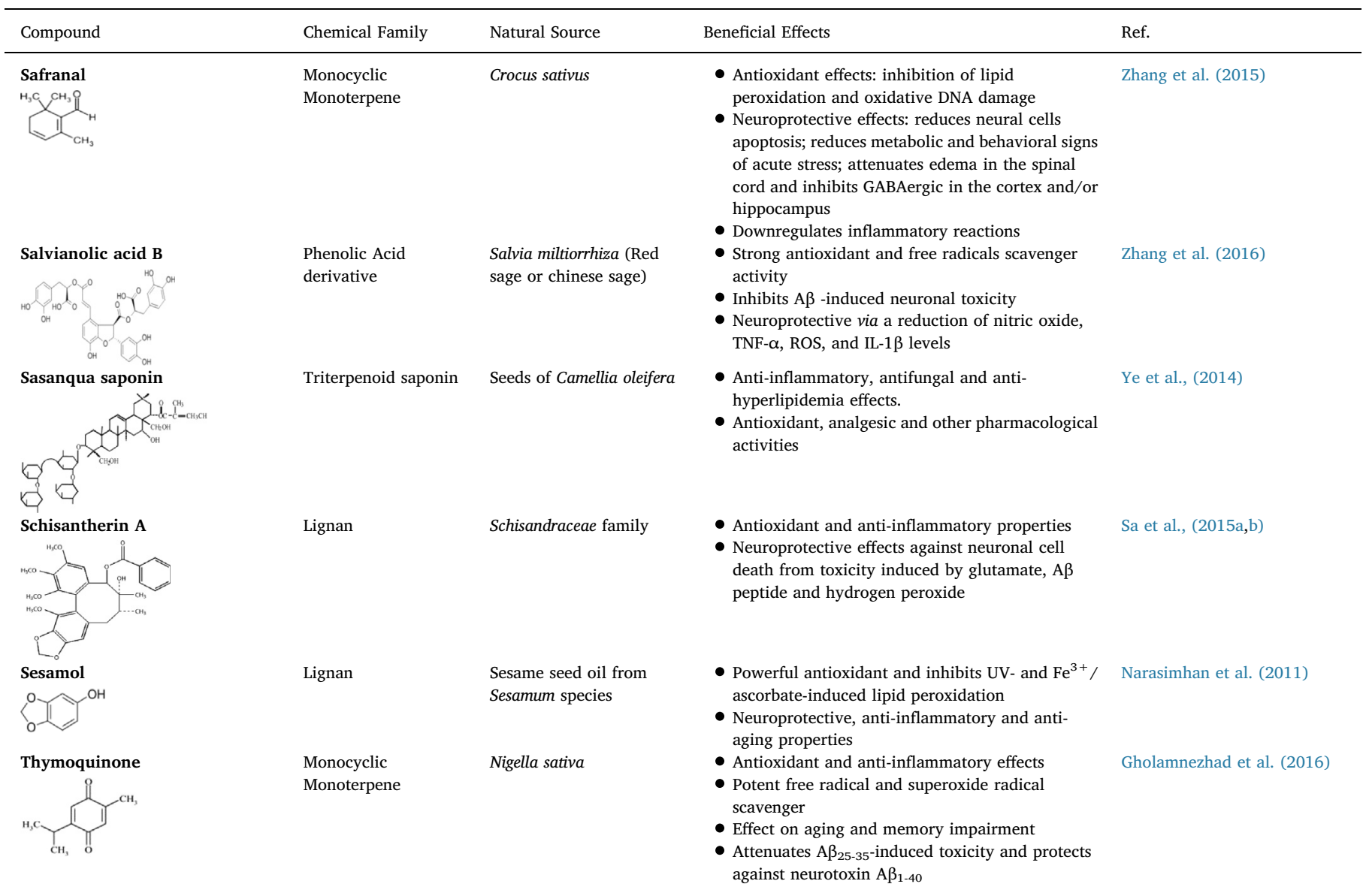

Aß: Amyloid $\beta$-peptide ; AD: Alzheimer disease; CIR: Cerebral Ischemia Reperfusion; COX-2: Cyclooxygenase-2; EGCG: Epigallocatechin gallate; GABA: $\gamma$ Aminobutyric acid NFkB: Nuclear factor кB; NFT: Neurofibrillary tangles; PD: Parkinson disease; ROS: Reactive Oxygen Species; TNF- $\alpha$ : Tumor necrosis factor $\alpha$; UV: Ultraviolet radiation.

A summary of the most reported natural compounds with neuroprotection effects is presented in Table 1.

\section{Challenges in the administration of natural compounds for neuroprotection}

\subsection{Low bioavailability and poor pharmacokinetic profiles}

The brain is very vulnerable to oxidative stress and recently, bioactives with anti-inflammatory and antioxidant effects have gained relevance on the prevention of the damages caused by free radicals and also on the treatment of ARND (Albarracin et al., 2012; Aquilano et al., 2008). Many of bioactive antioxidant and anti-inflammatory agents are provided by food and several studies show that diets rich on these compounds play an important role on ARND prevention, however their beneficial effect is greatly affected by their low bioavailability and poor pharmacokinetic profile (Albarracin et al., 2012; Manach, 2004).

For decades, the administration of drugs into a person has been accomplished via various routes: oral, parenteral, topical, and inhalational, among others. Of all the routes, oral administration is the major conventional and well accepted method for drug delivery. However, oral administration has several problems such as variable absorption, fast metabolism, and poor bioavailability mostly because of $\mathrm{pH}$ variation conditions and the digestion of the administrated actives due to the presence of proteolytic enzymes in the gastrointestinal tract (Lúcio et al., 2010). To be efficiently absorbed from the digestive tract, an element has to cross the enterocytes plasma membrane that covers the gut lumen. Most of the compounds cross this membrane through passive diffusion via the lipid bilayer, but for that being possible it is essential that the bioactives possess the required balance of lipophilicity/ hydrophilicity (Lúcio et al., 2010). After passing the absorption obstacle and reaching circulation, the bioactive redistributes throughout the body. However, the redistribution can be hindered by a number of obstacles. The hydrophobic actives are often extensively bound to plasma proteins to be able to be transported in blood. As a consequence the bioactives may be accumulated in adipocytes or rapidly change in the liver into hydrophilic metabolites and be removed through urine or excreted into the bile (Lúcio et al., 2010).

In summary, due to the poor solubility and permeability, instability or even the biotransformation that happens before and after bioactives enter systemic circulation it is difficult for these compounds to reach the brain (Ratnam et al., 2006). Ultimately, even if a bioactive for ARND treatment is able to be absorbed and distributed in the body long enough to reach the brain, it still has to overcome other blood-tissue barriers like the blood-brain barrier (BBB) (Lúcio et al., 2010). Hence, one of the challenges nowadays is to create NC that are able to deliver bioactives directly to the brain overcoming the biological barriers found by these compounds (Alam et al., 2010).

\subsection{Overcoming blood brain barrier - a major challenge}

The treatment of neurological diseases is not an easy task, especially due to the brain physical barriers like the BBB. It is the crucial barrier when we are dealing with the transport of compounds from blood into 


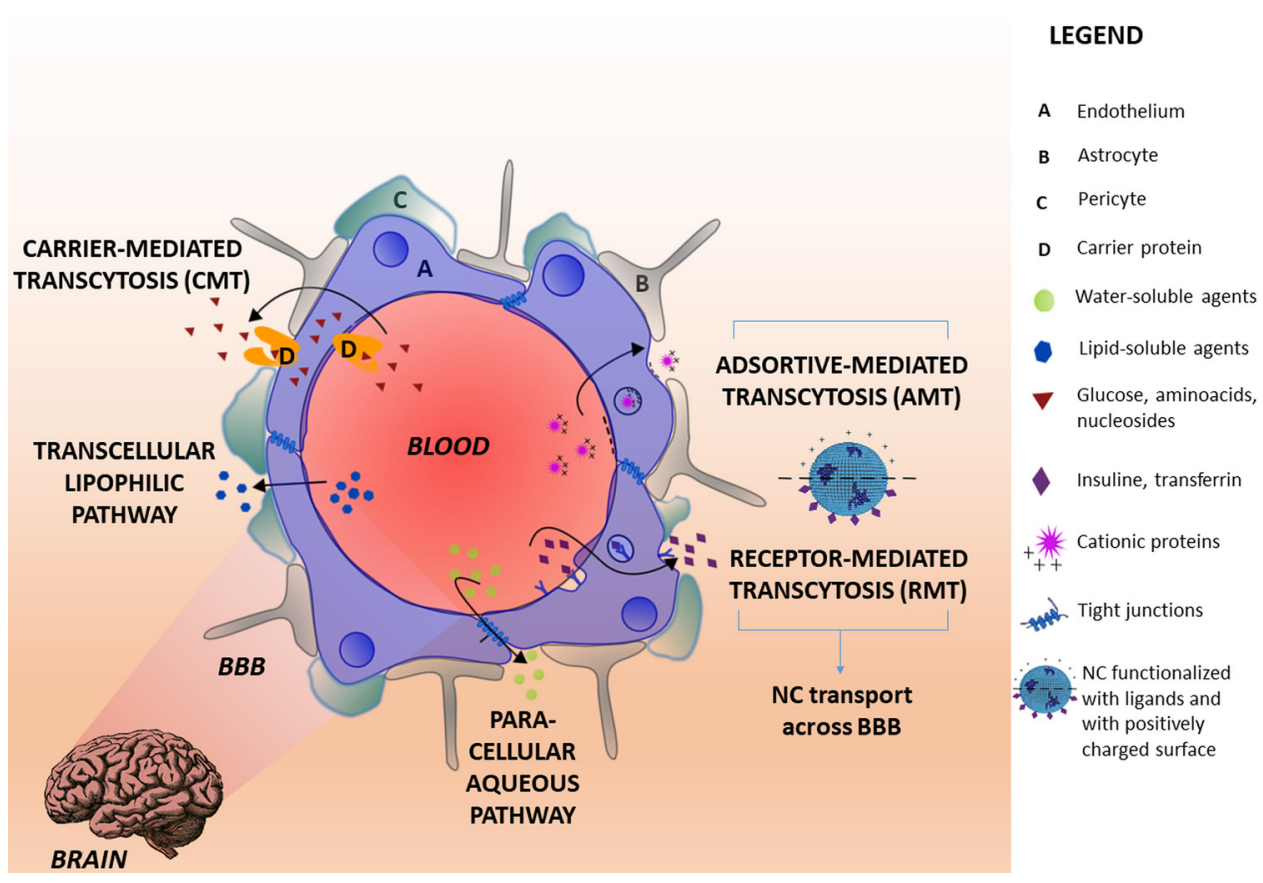

Fig. 2. Transport pathways across Blood Brain Barrier (BBB).

The main routes for molecular traffic across the $\mathrm{BBB}$ are shown as well as the type of solutes that use each pathway. Nanocarriers (NC) preferential pathways are (i) receptor mediated transcytosis (RMT) when the NC surface is functionalized with ligands recognized BBB receptors and (iii) adsorptive-mediated transcytosis (AMT) when the NC surface is positively charged.

brain (Fig. 2), providing the protection of the CNS (Chen and Liu, 2012; Gaillard et al., 2012).

BBB is made up of endothelial cells without fenestrations characterized by the presence of tight junctions, which form a continuous cellular membrane that restricts the transport of molecules. This diffusion barrier keeps the CNS homeostasis by transporting the required nutrients to the brain for its normal functions (Cardoso et al., 2010; Lun et al., 2012). BBB is also composed by pericytes and astrocytes. The pericytes are essential for the structural support. These cells are also associated with transport across the BBB and in the vessels' differentiation, as well as in formation of endothelial tight junctions (Ballabh et al., 2004; Cardoso et al., 2010). Astrocytes are glial cells and are important for the normal neuronal function (Cardoso et al., 2010).

BBB is not only a protective barrier, but also a transport barrier that regulates and provides several routes for the transport of molecules to the brain. Substance diffusion into the brain can be classified into paracellular (transport of ions and solutes between adjacent cells through the tight junctions and according to their concentration) and transcellular (transport of solutes through the cells) (Cardoso et al., 2010). When it comes to the transcellular pathway it involves different mechanisms such as: transport made by carrier proteins (carriermediated transport; CMT); specific receptor-mediated mechanisms (receptor mediated transcytosis; RMT) or vesicular mechanisms (adsorptive mediated transcytosis, AMT). CMT allows little molecules (like glucose or amino acids) binding to specific membrane protein carriers (Alam et al., 2010; Chen and Liu, 2012). RMT mechanism consists in the transport of macromolecules such as transferrin, insulin, or leptin, and it is mediated by specific receptors present on the endothelial cells (Chen and Liu, 2012; Pardridge, 2003). AMT mechanism of transport through BBB is initiated due to an electrostatic interaction that occurs between a plasma membrane that is negatively charged and a substance that is positively charged (Chen and Liu, 2012).

Although there are several routes to cross BBB, the molecules that can pass it by passive diffusion must possess very specific characteristics like small size and endothelial permeability, which are very hard to fulfil. Therefore, it was already proved that $98 \%$ of neurotherapeutic molecules cannot pass across the BBB (Zlokovic, 2008). Circumventing BBB could thus be achieved through the administration of nanodelivery systems that can target and control the release of different bioactive agents that are used to treat ARND.

\section{Nanocarriers as an emerging platform for drug delivery to brain}

There are significant challenges when treating CNS disorders and a possible solution to these difficulties is the use of Nanotechnology. The possibility of using NC with advantageous characteristics, like high biological and chemical stability, possibility of integrating both hydrophobic and hydrophilic drugs, biologicals (enzymes, peptides or nucleic acids) or other bioactive compounds, and the possibility of being administered using different ways (including inhalational, oral, and parenteral) makes NC attractive for medical applications (MartinBanderas et al., 2011). NC systems for the delivery of bioactive compounds to treat ARND possess additional features over classical therapies: (i) the possibility to encapsulate different payloads with synergic effects that are efficient at multiple etiological targets (Silva, 2006; Spuch et al., 2012); (ii) the power to attain specific biodistribution profiles that were not possible before microscale delivery systems or with purely molecular (Etheridge et al., 2013) and the possibility to functionalize NC surface with targeting ligands that make use of BBB transport routes for effective delivery of bioactives to the brain (Alam et al., 2010; Tosi et al., 2016).

NC design strategies of stealth, targeting and triggering have been developed for their numerous advantages: sustained and local delivery of bioactives to provide a higher therapeutic effect; reduce toxicity while able to maintain the therapeutic effects; biocompatibility and greater safety; reduction in high-dose-associated side effects and adjustable/controlled bioactive delivery rate that increase the duration of effect (Jong and Borm, 2008; Kim et al., 2009; Kumar et al., 2014). These strategies (Fig. 3) are particularly important for the rational design of NC aiming the delivery of bioactive compounds to treat ARND.

\subsection{Stealth strategies}

When in blood circulation, the immune system easily recognizes NC and clear them with phagocytes thereby reducing their chances to reach target tissues. Stealth strategies consist on coating NC surface with hydrophilic chains (e.g. polyethylene glycol, PEG) to enhance the stability of NC against aggregation. Such modification (named as "PEGylation") reduces opsonisation of NC in plasma and decreases its recognition and removal by the mononuclear phagocytes (dendritic cells, monocytes, and macrophages) (Kabanov and Gendelman, 2007). 


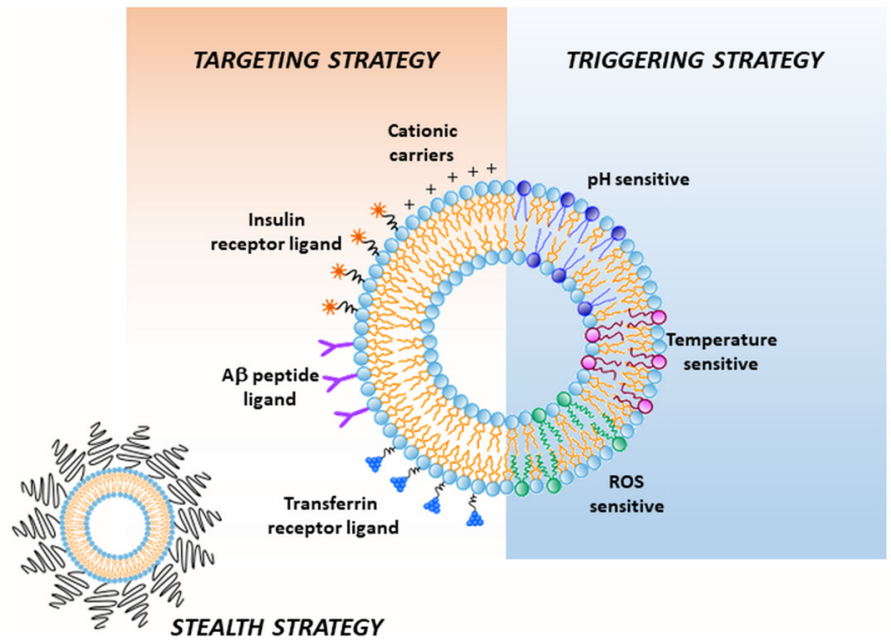

5.2. Targeting strategies

There are two types of targeting strategies that maybe used when designing NC for delivery of therapeutics: passive targeting and active targeting strategies.

\subsubsection{Passive targeting}

Passive targeting strategies are the physical properties of NC (e.g. size and surface charge) that we can control to enhance their delivery to a specific target tissue. The range of $2-200 \mathrm{~nm}$ is the NC size that is desirable for systemic administration due to therapeutic reasons (Jo et al., 2015). Nevertheless, NC smaller than $5 \mathrm{~nm}$ are at the risk of renal excretion: even if they escape renal excretion, they suffer rapid clearance from target tissues. Therefore sizes superior to $5 \mathrm{~nm}$ are preferable to reach higher circulation times (Jo et al., 2015). In the development of nanotherapeutics against CNS diseases, NC of $20-100 \mathrm{~nm}$, have proved to be big enough to avoid renal glomerular filtration and small enough to pass through BBB (Jo et al., 2015). Larger NC will not passively overcome BBB and will require active targeting strategies (described below). NC upper size limit is thought to be around $200 \mathrm{~nm}$, as NC larger than this size are scavenged by reticuloendothelial systems (RES), chiefly the liver's Kupffer cells and the spleen's macrophages. Bigger particles that are larger than $2 \mu \mathrm{m}$ are also caught by the pulmonary capillary vessels (Jo et al., 2015).

In addition, NC surface charge modulates biodistribution, interaction with biological environments, and cellular uptake. In general, NC that are positively charged are acknowledged as internalized more easily than NC that are negatively and neutral charged (Jo et al., 2015). In the case of BBB targeting, a positive surface charge is more advantageous as it will favor NC uptake by AMT transport (Fig. 3).

\subsubsection{Active targeting}

Active targeting strategies consist on NC surface functionalization with ligands (for example, monoclonal antibodies, sugar moieties, peptide fragments, or other ligands that partake in recognizing receptors) that bind to signal receptors at the target tissues. These strategies favor NC targeted delivery to given areas, while avoiding accumulating bioactives in nontargeted tissues.

BBB is impermeable for most of the NC, therefore targeting strategies are essential when developing brain delivery systems. Moreover, targeting moieties bounded to the NC surface (Fig. 3) will facilitate the specific delivery of therapy to brain while avoiding toxic off-target effects resultant from NC accumulation in healthy tissues. Among the transport mechanisms across BBB, RMT and AMT are the ones with most potential for NC brain transcytotic uptake (Fig. 2). Table 2 summarizes the reported strategies for targeting NCs to BBB using these two

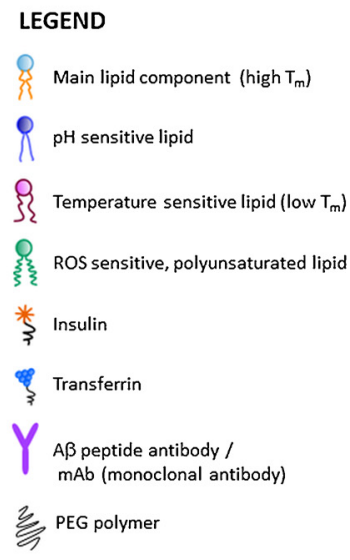

Fig. 3. Nanocarriers design strategies.

Targeting strategies for BBB include nanocarriers surface functionalization with: ligands (e.g: insulin and transferrin) that target receptors of the $\mathrm{BBB}$; monoclonal antibodies $(\mathrm{mAb})$ that target $\mathrm{A} \beta$ peptide receptors or other receptors at $\mathrm{BBB}$ and cationic carriers that target negatively charged BBB endothelium. Triggering strategies include lipids sensitive to several stimuli $(\mathrm{pH}$, temperature and reactive oxygen species, ROS). Stealth strategies are normally surface functionalization with poly (ethylene) glycol (PEG).

Table 2

Targeting strategies for transport of NC across BBB.

\begin{tabular}{cll}
\hline BBB Transport system & Receptors/Target & Targeting ligand strategy \\
\hline Receptor mediated & InsR & Ins; InsR mAb \\
transcytosis & IGF1R, IGF2R & IGF \\
(RMT) & TfR & Tf; TfR mAb \\
& LepR & Lep \\
& SR-B1 or AceLDLR & LDL, LN \\
& LDLR and LRP & Apolipoproteins PS80 \\
& GpR & Man \\
& A $\beta$ peptide & A $\beta$ mAb \\
Adsortive mediated & Negative & Cationic ligands (CPP like TAT \\
transcytosis & glycoprotein on & derived peptides; cationic \\
(AMT) & endothelial capillary & proteins; cationic lipids) \\
Adjuvant mediated & BBB cellular & PS80, ethanol, DMSO, SDS \\
BBB disruption & membrane &
\end{tabular}

AceLDLR: Acetylated LDL receptor; A $\beta$ peptide: Amyloid $\beta$-peptide; CPP: Cell penetrating peptides; DMSO: Dimethilsulfoxide; GpR: Glucoprotein receptor; IGF: Insulin-like growth factor; IGF1R and IGF2R: Insulin-like growth factor receptors; Ins: Insulin; InsR: Insulin receptor; LDL: Low-density lipoprotein; LDLR: Low-density lipoprotein receptor; Lep: Leptin; LepR: Leptin receptor; LN: Lipid nanoparticle; LRP: Low-density lipoprotein receptor related protein; mAb: Monoclonal Antibody; Man: Mannose; PS80: Polysorbate or Tween 80; SDS: Sodium lauril sulfate; SR-B1: Scavenger receptor type B1; TAT: CPP derived from the HIV trans-activator of transcription; Tf: Transferrin; TfR: Transferrin receptor.

transport mechanisms.

RMT transport is the most common pathway for NC uptake into brain and consists of: (i) interaction of the NC surface ligands with specific receptors that are expressed on BBB (targeting ligand strategies mentioned on Table 2); (ii) formation of endocytotic-vesicles enveloping NC; (iii) transcytosis across the BBB; and (iv) exocytosis of NC in CNS parenchyma.

Lipid NC have additional advantages over other NC (polymeric or inorganic, for instance) regarding their capacity to overcome BBB, as lipid nanoparticles are thought to mimic LDL particles and subsequently, the LDL receptor transports them, even when they are not decorated at the surface with targeting ligands (Alam et al., 2010).

AMT transport depends on the electrostatic interaction of ligand of the NC surface that is positively charged with the luminal surface of endothelial cells that have negative charges. For a more extensive reading on $\mathrm{BBB}$ targeting strategies, please refer to reviews on the subject (Alam et al., 2010; Tosi et al., 2016).

Other strategies of targeting and/or increasing the permeability of the BBB vascular endothelium consist on NC surface functionalization (e.g. with PS80 and SDS) or incorporation of adjuvants (e.g. DMSO, 
ethanol) in the NC composition (Table 2).

\subsection{Triggering strategies}

Triggering strategies (Fig. 3) consist on using NC containing stimuliresponsive moiety to trigger the release of bioactives (e.g. $\mathrm{pH}$, temperature or enzymatic triggering). Bioactives' release triggered by a $\mathrm{pH}$ decrease has been used to improve delivery of encapsulated therapies to the more acidic tissues (e.g. inflamed or cancer tissues) and thus can be used as a strategy for ARND with associated neuroinflammation. Furthermore pH-responsive systems can be applied for external and internal (endosomes) cellular release. High levels of ROS produced in ARND can also be used as triggering strategies on NC that are labile upon oxidative conditions inducing their cargo release. For a more complete description over stimuli-responsive triggering strategies, please refer to a review on the subject (Mura et al., 2013).

\section{Lipid nanocarriers for the treatment of age related diseases}

Lipid-based NC are frequently chosen over other NC as they mimic in many ways the natural lipid environment found on biomembranes and often present lower toxicity when applied in vivo (Pujara, 2012). Furthermore, lipid based NC are able to reach the brain using physiological transport mechanisms, like the LDL receptor pathway (Alam et al., 2010).

Examples of lipid based NC used for ARND treatments are: liposomes; micelles; nanoemulsions such as self-nanoemulsifying drug delivery systems (SNEDDS) and self-double-emulsifying drug delivery system (SDEDDS); lipid NC with solid matrix (SLN and NLC) and ethosomes. These different types of lipid based NC are represented in
Fig. 4 and will be briefly described.

\subsection{Liposomes}

Liposomes are formed by amphiphiles (e.g. phospholipids) that, once dispersed in an aqueous media, self-assemble as vesicles (Lúcio et al., 2010). This vesicles are composed by lipid bilayers, where the hydrophobic part of the amphiphiles (hydrophobic fatty acid chains) will be oriented away from the aqueous polar phase, whereas the molecules' polar portions (head group region) are exposed to the aqueous solvent that exists in the vesicle core and also surrounding the vesicle (Lúcio et al., 2010) (Fig. 4).

The use of liposomes as NC for drug delivery, has been focused on the production of three types of liposomes: multilamellar vesicles (MLV), composed by several lipid bilayers approximately concentric; large unilamellar vesicles (LUV), that have a size range comprised between 100 and $500 \mathrm{~nm}$; and small unilamellar vesicles (SUVs) smaller than $100 \mathrm{~nm}$ (Allen and Cullis, 2013). Nonetheless, the properties of liposomes are extremely adaptable, changing with size, preparation method and lipid composition, which determines the strength and charge of the liposomal surface (Allen and Cullis, 2013). The tunable nature of liposomes increase its potential to achieve high encapsulation of active compounds.

There are numerous liposomal preparation methods, including: (i) lipid film hydration method followed by mechanical approaches such as homogenization techniques (e.g sonication, microfluidization, extrusion); (ii) approaches based in the replacement of organic solvents by aqueous solutions (e.g. ethanol injection); (iii) reverse phase evaporation techniques and (iv) techniques based in detergent removal. The selection of the applied method is intrinsically connected with the

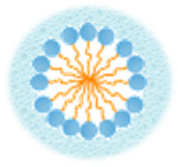

MICELLES

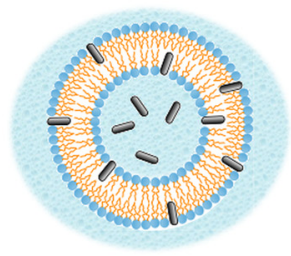

ETHOSOMES

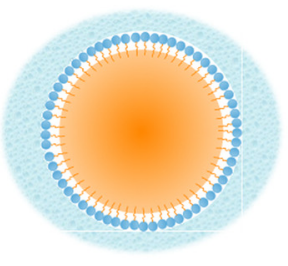

NANOEMULSIONS (SNEDDS)

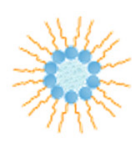

INVERTED MICELLES

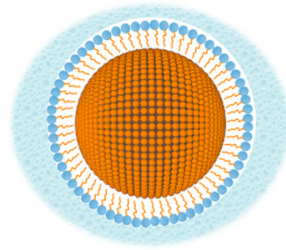

SLN

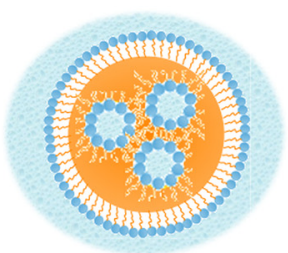

SDEDDS (W/O/W)

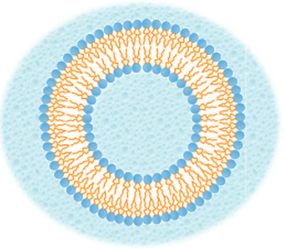

LIPOSOMES

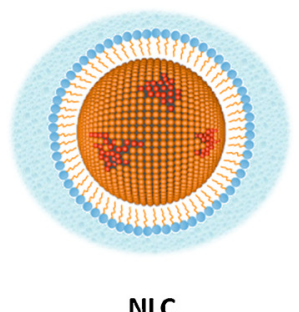

NLC

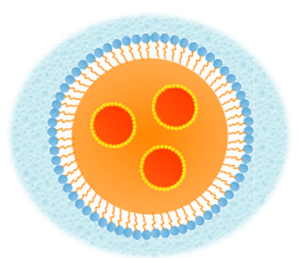

Lipophilic stabilizer

Aqueous phase

Fig. 4. Types of lipid based nanocarriers.

SLN: Solid lipid nanoparticles; NLC: Nanostructured lipid carriers; SNEDDS: Self-nanoemulsion drug delivery systems; SDEDDS: Self-double emulsion drug delivery systems; O: oil phase; W: water phase. 
following parameters: (i) properties of the constituent lipids and of the encapsulated compounds; (ii) nature of the medium in which lipid vesicles will be dispersed; and (iii) therapeutical application. Along with these parameters, it is perceptible that most of the liposomal preparation methods are inadequate for large scale production and that the ethanol injection and the lipid film hydration method followed by extrusion are the most frequently used (Allen and Cullis, 2013; Lúcio et al., 2010).

\subsection{Lipid nanocarriers with solid matrix: solid lipid nanoparticles (SLN)} and nanostructured lipid carriers (NLC)

Over time, lipid NC with solid matrix have increased its popularity regarding the transport of low-aqueous soluble drugs (Pardeike et al., 2009; Üner and Yener, 2007). In comparison with liposomes, these NC present advantages like the higher protection of incorporated active compounds and an easy scale up potential (Üner and Yener, 2007).

These NC can be prepared by different methods described in the literature: ultrasonication, microemulsion, emulsification-solvent evaporation, phase inversion, solvent displacement, emulsifalsoicationsolvent diffusion, and the most used owing to its scale up potential high pressure homogenization technique (Pardeike et al., 2009; Üner and Yener, 2007). However, all of these methods are based on solid lipid or lipid blends (mixture of solid and liquid lipid) fusion. Briefly, the lipid phase is melted being posteriorly dispersed through high-speed agitation in an aqueous solution of equivalent temperature containing a tensioactive or a stabilizer. Afterwards, the obtained pre-emulsion is homogenized, recurring to a high-pressure homogenizer, achieving an oil/water nanoemulsion. At last, emulsion droplets crystalize forming lipid NC with a solid matrix (if all lipid components are solid) also known as Solid Lipid Nanoparticles (SLN) or forming NC with a matrix containing solid and liquid lipids designated as Nanostructured Lipid Carriers (NLC) (Pardeike et al., 2009; Üner and Yener, 2007). Thus, these NC present a hydrophobic matrix covered by a tensioactive layer that favors their dispersion in water (Fig. 4). The active compound is dissolved during the fusion step and before the high-shearing step either in the fluid lipid phase or in the aqueous phase, according to its chemical nature. Depending on the position and distribution of the active compound it is possible to distinguish three incorporation models both at SLN or NLC: the model of homogenous matrix that presents the actives distributed in the lipid matrix; the model of enriched shell where the therapeutic agent is at the surface of the NC; and, the model of enriched nucleus where actives possess a nuclear location and are surrounded by lipids with a protective purpose (Pardeike et al., 2009; Üner and Yener, 2007).

Despite their similarities in preparation and active compounds incorporation, there are important differences between SLN and NLC. SLN are predominantly composed by saturated monoacid triglycerides and hard fats, which are solid at body and room temperature, and that enables mobility reduction of the incorporated active compounds, preventing their release from the carrier (Pardeike et al., 2009; Üner and Yener, 2007). Contrastingly to highly ordered SLN, NLC possess a less ordered solid matrix which demonstrates a superior charge capacity of active compounds, minimizing their undesirable expulsion during storage (Pardeike et al., 2009). NLC can be classified as: imperfect crystalline structure NLC, obtained through a mixture of solid lipids with low quantities of liquid lipid; amorphous NLC, composed by a mixture of solid lipids with lipids that are unable to recrystallize after homogenization; and, finally, multiple NLC, formed by a mixture of full or medium chain solid lipids (Üner and Yener, 2007).

\subsection{Ethosomes}

Although classic liposomes are outstanding candidates for drug delivery, their ability to infiltrate the skin is still reduced, decreasing its potential for transdermal drug delivery. In this way, ethosomes were developed as lipid vesicular systems composed by: phospholipids; ethanol (known as an efficient permeability enhancer) in high quantities (20-45\%), and water. This ethosomal system is known to improve transdermal drug delivery, both in vitro and in vivo. The mode of action of ethosomes is based on the interaction of ethanol with lipid molecules that are located in the polar head group region, which increases the fluidity of the lipid membrane. The ethanol intercalation may furnish the vesicles with soft flexible properties that makes it possible for penetration of deeper skin layers. The same rationale can be applied to brain delivery as ethosomes characteristics might be useful to promote BBB permeabilization and delivery of bioactives to the brain (Table 2).

Considering their production, these vesicular systems are prepared in a closed recipient through the dissolution of the drug and lipids in ethanol, with the slowly addition and fine flow of the aqueous component at a constant rate and under constant mixing.

In comparison with liposomes, ethosomes similarly hold the advantage of presenting small dimensions due to the negative net charge in vesicle surface induced by the presence of ethanol. Furthermore, these novel phospholipid vesicular carriers seem to be capable of carrying in an efficient manner compounds of different lipophilicities (Godin and Touitou, 2003).

\subsection{Micelles}

Micelles are self-assembly aggregates of surface-active agents (surfactants) that occur above a concentration that is well-defined and is called the critical micelle concentration (CMC). The structure of the surfactants' hydrophobic tails and head groups included in micelles composition controls their packing parameter (PP), thereby controlling the physical properties of the micelles, viz. the CMC and their aggregation number. In aqueous bulk media, inverted conical shaped surfactants $(\mathrm{PP}<1)$ aggregate in a single lipid layer rather than bilayers: the surfactants' hydrophobic part that forms the micelle is oriented in the cluster and located away from the aqueous polar phase, whereas the molecule polar parts (head groups) are exposed to the solvent (Fig. 4). In a reduced aqueous content, conical shaped surfactants $(\mathrm{PP}>1)$ can aggregate in an inverted structure (inverted micelles) (Fig. 4). In both cases, micelles and inverted micelles possess polar and apolar regions and can be used as simple delivery systems able to carry hydrophobic and hydrophilic bioactive compounds (Torchilin, 2006).

\subsection{Nanoemulsions}

Nanoemulsions are colloidal dispersions that can be produced from oil, water, and surfactants, and originate small emulsion droplets with sizes of $10-100 \mathrm{~nm}$. Normally, nanoemulsions can be assorted as bicontinuous, water-in-oil (W/O) and oil-in-water $(\mathrm{O} / \mathrm{W})$. Also, the process used to prepare nanoemulsions as well as their composition and the environmental conditions are responsible to define their structure, creating a variety of different systems (Date et al., 2010; McClements, 2012).

Nanoemulsions are widely used as delivery systems due to their advantages: i) ability to solubilize hydrophilic and hydrophobic agents; ii) improved stability of agents; iii) greater esthetic appeal and skin feel when administrated as transdermal formulations; iv) improved dermal and mucosal transport; v) improved oral bioavailability; vi) flow properties ranging from liquid to highly solid; vii) optical properties fluctuating from opaque to nearly transparent and viii) easy manufacture and scale up (Date et al., 2010; McClements, 2012). Despite the numerous advantages of nanoemulsions, these delivery systems are thermodynamically unstable requiring a specific design to reach a satisfactory kinetic stability (Date et al., 2010; McClements, 2012).

In respect to the methods used for nanoemulsions fabrication, they can be separated into low-energy emulsification methods or high-energy emulsification methods. The first, low-energy emulsification 
Table 3

Lipid nanocarriers containing natural compounds (Barras et al., 2009; Campos et al., 2014; Neves et al., 2013; Ramachandran and Thangarajan, 2016; Vanaja et al., 2013; Wang et al., 2011).

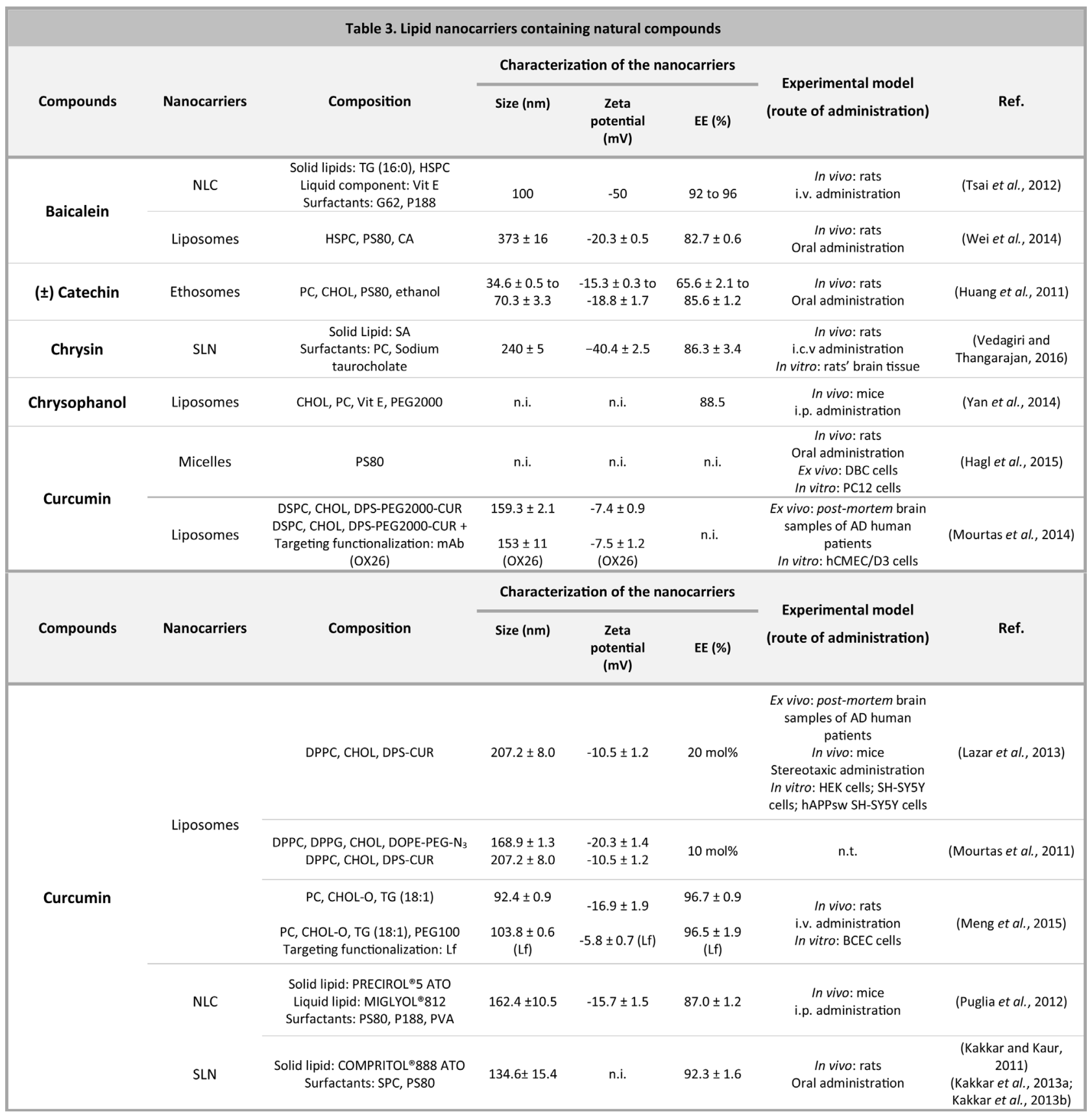

(continued on next page) 
Table 3 (continued)

\begin{tabular}{|c|c|c|c|c|c|c|c|}
\hline \multirow[b]{2}{*}{ Compounds } & \multirow[b]{2}{*}{ Nanocarriers } & \multirow[b]{2}{*}{ Composition } & \multicolumn{3}{|c|}{ Characterization of the nanocarriers } & \multirow{2}{*}{$\begin{array}{c}\text { Experimental model } \\
\text { (route of administration) }\end{array}$} & \multirow[b]{2}{*}{ Ref. } \\
\hline & & & Size $(\mathbf{n m})$ & $\begin{array}{l}\text { Zeta } \\
\text { potential } \\
(\mathrm{mV})\end{array}$ & $\mathrm{EE}(\%)$ & & \\
\hline \multirow[b]{2}{*}{ Curcumin } & SLN & $\begin{array}{c}\text { Solid lipid: SA } \\
\text { Surfactants: PC, MYRJ } 52\end{array}$ & $\approx 60$ & -27 & n.i. & $\begin{array}{l}\text { In vivo: mice } \\
\text { i.p. administration }\end{array}$ & (Wang et al., 2015) \\
\hline & $\begin{array}{l}\text { Nanoemulsion } \\
\text { (SNEDDS) }\end{array}$ & $\begin{array}{l}\text { Oil phase: } \mathrm{CAPMUL}^{\oplus} \mathrm{MCM} \text {, } \\
\text { CAPTEX }{ }^{\oplus 500} \\
\text { Surfactants: } \mathrm{CREMOPHOR}^{\oplus} \mathrm{EL} ; \\
\text { PS80 } \\
\text { Co-surfactants: PEG400; } \\
\text { TRANSCUTOL } \\
\text { Surface coating: } \mathrm{CHS}\end{array}$ & $\begin{array}{c}35.8 \pm 2.6 \\
37.8 \pm 3.1 \\
(\mathrm{CHS})\end{array}$ & $\begin{array}{l}-19.6 \pm 1.9 \\
+27.2 \pm 2.1 \\
\quad(\mathrm{CHS})\end{array}$ & n.i. & $\begin{array}{l}\text { In vitro: SK-N-SH cells } \\
\text { Ex vivo: Sheep nasal } \\
\text { mucosa }\end{array}$ & (Sood et al., 2014) \\
\hline \multirow{2}{*}{ EGCG } & $\begin{array}{l}\text { Nanoemulsion } \\
\text { (SNEDDS) }\end{array}$ & $\begin{array}{l}\text { Oil phase: } \angle A B R A F A C \\
\text { Surfactants: } P 15 H, P C\end{array}$ & 57 & n.i. & 95 & n.t. & (Barras et al., 2009) \\
\hline & $\begin{array}{c}\text { Lipid } \\
\text { nanoparticles }\end{array}$ & Proprietary composition & 30 to 80 & n.i. & n.i. & $\begin{array}{l}\text { In vivo: rats } \\
\text { Oral administration }\end{array}$ & (Smith et al., 2010) \\
\hline Ligustrazine & Ethosomes & EPC, ethanol & $146.3 \pm 24.6$ & n.i. & $70.2 \pm 1.2$ & $\begin{array}{c}\text { In vivo: rats } \\
\text { Transdermal administration } \\
\text { In vitro: rats' abdominal } \\
\text { skin }\end{array}$ & (Shi et al., 2012) \\
\hline \multirow[b]{2}{*}{ Compounds } & \multirow[b]{2}{*}{ Nanocarriers } & \multirow[b]{2}{*}{ Composition } & \multicolumn{3}{|c|}{ Characterization of the nanocarriers } & & \\
\hline & & & Size $(\mathrm{nm})$ & $\begin{array}{l}\text { Zeta } \\
\text { potential } \\
(\mathrm{mV})\end{array}$ & EE (\%) & (route of administration) & Ref. \\
\hline \multirow[t]{2}{*}{ Luteolin } & Liposomes & SPC, CHOL, Vit E & $150 \pm 25$ & n.i. & 98 & $\begin{array}{c}\text { In vivo: rats } \\
\text { i.p. administration } \\
\text { In vitro: primary rats' } \\
\text { neurons }\end{array}$ & (Zhao et al., 2011) \\
\hline & SLN & $\begin{array}{c}\text { Solid Lipid: GMS } \\
\text { Surfactants: SPC, PS80 }\end{array}$ & 47 to 118 & -9.6 & $74.8 \pm 1.4$ & $\begin{array}{l}\text { In vivo: rats } \\
\text { Oral administration }\end{array}$ & (Dang et al., 2014) \\
\hline Piperine & SLN & $\begin{array}{l}\text { Solid Lipid: GMS } \\
\text { Surfactants: PC, PS20, PS80 } \\
\text { Surface coating: PS80 }\end{array}$ & $\begin{array}{c}258.0 \pm 5.5 \\
\text { to } \\
277.6 \pm 2.4\end{array}$ & $\begin{array}{l}-44.3 \pm 1.2 \\
\text { to } \\
-45.0 \pm 1.7\end{array}$ & $\begin{array}{l}72.0 \\
\text { to } 68.2\end{array}$ & $\begin{array}{c}\text { In vivo: rats } \\
\text { i.p. administration } \\
\text { In vitro: rats' brain tissue }\end{array}$ & (Yusuf et al., 2013) \\
\hline \multirow{5}{*}{ Quercetin } & \multirow{4}{*}{ Liposomes } & PC & n.i. & n.i. & n.i. & $\begin{array}{l}\text { In vivo: rats } \\
\text { i.v. administration }\end{array}$ & (Rivera et al., 2008) \\
\hline & & $\begin{array}{c}\text { EPC, CHOL } \\
\text { EPC, CHOL, PEG400 }\end{array}$ & n.i. & n.i. & n.i. & $\begin{array}{l}\text { In vivo: rats } \\
\text { Intranasal administration }\end{array}$ & $\begin{array}{l}\text { (Phachonpai et al., } \\
\text { 2010) } \\
\text { (Tong-un et al., 2010) }\end{array}$ \\
\hline & & $\mathrm{PC}, \mathrm{CHOL}$ and $\beta-\mathrm{CD}$ & n.i. & n.i. & n.i. & $\begin{array}{l}\text { In vivo: Newborn piglets } \\
\text { i.v. administration }\end{array}$ & (Blasina et al., 2015) \\
\hline & & $\begin{array}{c}\mathrm{PE}, \mathrm{CHOL}, \mathrm{DCP} \\
\text { Targeting functionalization: MAN }\end{array}$ & 50 & n.i. & n.i. & $\begin{array}{l}\text { In vivo: rats } \\
\text { i.v. administration }\end{array}$ & (Sarkar and Das, 2006) \\
\hline & SLN & $\begin{array}{c}\text { Solid Lipid: COMPRITOL }{ }^{\oplus 888} \text { ATO } \\
\text { Surfactant: PS80 }\end{array}$ & 159 & +21.1 & 85.7 & $\begin{array}{l}\text { In vivo: rats } \\
\text { i.v. administration }\end{array}$ & (Dhawan et al., 2011) \\
\hline
\end{tabular}

(continued on next page) 
Table 3 (continued)

\begin{tabular}{|c|c|c|c|c|c|c|c|}
\hline \multirow[b]{2}{*}{ Compounds } & \multirow[b]{2}{*}{ Nanocarriers } & \multirow[b]{2}{*}{ Composition } & \multicolumn{3}{|c|}{ Characterization of the nanocarriers } & \multirow{2}{*}{$\begin{array}{c}\text { Experimental model } \\
\text { (route of administration) }\end{array}$} & \multirow[b]{2}{*}{ Ref. } \\
\hline & & & Size (nm) & $\begin{array}{l}\text { Zeta } \\
\text { potential } \\
(\mathrm{mV})\end{array}$ & EE (\%) & & \\
\hline \multirow{3}{*}{ Quercetin } & SLN & $\begin{array}{c}\text { Solid Lipid: } \text { COMPRITOL }^{\otimes} 888 \text { ATO } \\
\text { Surfactant: PC }\end{array}$ & $74.6 \pm 4.2$ & $-7.7 \pm 0.4$ & \multirow[b]{2}{*}{$>80$} & \multirow{2}{*}{$\begin{array}{l}\text { In vivo: rats } \\
\text { Oral administration } \\
\text { In vitro: Caco- } 2 \text { cells and } \\
\text { rats' brain tissue }\end{array}$} & \multirow{2}{*}{ (Kumar et al., 2016) } \\
\hline & NLC & $\begin{array}{l}\text { Solid Lipid: } \text { COMPRITOL }^{\circledR} 888 \text { ATO } \\
\text { Liquid Lipid: Vit E } \\
\text { Surfactant: PC }\end{array}$ & $67.5 \pm 3.3$ & $-8.9 \pm 0.5$ & & & \\
\hline & $\begin{array}{l}\text { Nanoemulsions } \\
\text { (SNEDDS) }\end{array}$ & $\begin{array}{c}\text { Oil phase: } \text { CO } \\
\text { Surfactants: } P C, \text { PEG15H }\end{array}$ & $19.3 \pm 0.2$ & $-5.3 \pm 0.5$ & 98.4 & $\begin{array}{c}\text { In vivo: rats } \\
\text { i.p. administration } \\
\text { In vitro: rats' brain tissue }\end{array}$ & (Galho et al., 2016) \\
\hline \multirow{3}{*}{ Resveratrol } & \multirow{2}{*}{ Liposomes } & HSPC, CHOL, DCP & $\begin{array}{l}134.2 \pm 0.3 \\
\text { to } \\
103.3 \pm 1.7\end{array}$ & -37 to -61 & $>80$ & $\begin{array}{l}\text { In vitro: blood leukocytes } \\
\text { and HUVEC cells }\end{array}$ & (Vanaja et al., 2013) \\
\hline & & $\mathrm{SPC}, \mathrm{CHOL}$ & 146 to 585 & n.i. & 93.5 & $\begin{array}{c}\text { In vivo: rats } \\
\text { Oral administration } \\
\text { In vitro: rats' nigral cells }\end{array}$ & (Wang et al., 2011) \\
\hline & SLN & $\begin{array}{l}\text { Solid Lipid: Cetyl palmitate } \\
\text { Surfactant: PS80 } \\
\text { Targeting functionalization: } \\
\text { DSPE-PEG-Avidine or Palmitate- } \\
\text { Avidine + biotinylated ApoE }\end{array}$ & $\begin{array}{l}167.8 \pm 19.9 \\
\text { to } \\
217.1 \pm 5.8\end{array}$ & $\begin{array}{l}-13.1 \pm 4.1 \\
\text { to } \\
-13.5 \pm 1.6\end{array}$ & $\begin{array}{l}98.0 \pm 2.5 \\
\text { to } \\
98.9 \pm 0.6\end{array}$ & In vitro: $\mathrm{hCMEC/D3}$ cells & (Neves et al., 2016) \\
\hline \multirow[b]{2}{*}{ Compounds } & \multirow[b]{2}{*}{ Nanocarriers } & \multirow[b]{2}{*}{ Composition } & \multicolumn{3}{|c|}{ Characterization of the nanocarriers } & & \\
\hline & & & Size (nm) & $\begin{array}{l}\text { Zeta } \\
\text { potential } \\
(\mathrm{mV})\end{array}$ & EE (\%) & (route of administration) & Ref. \\
\hline \multirow{3}{*}{ Resveratrol } & \multirow[b]{2}{*}{ NLC } & $\begin{array}{l}\text { Solid Lipid: Cetyl palmitate } \\
\text { Surfactant: PS80 } \\
\text { Targeting functionalization: } \\
\text { DSPE-PEG(2000) maleimide + } \\
\text { mAb (OX26, LB509) }\end{array}$ & $\begin{array}{c}249 \pm 1 \\
\text { to } \\
254 \pm 17\end{array}$ & $-5.0 \pm 0.1$ & $\begin{array}{l}75 \pm 7 \\
\text { to } \\
97 \pm 2\end{array}$ & In vitro: HBLEC cells & (Loureiro et al., 2017) \\
\hline & & $\begin{array}{l}\text { Solid Lipid: Cetyl palmitate } \\
\quad \text { Surfactant: PS60 } \\
\text { Solid Lipid: Cetyl palmitate } \\
\text { Liquid Lipid: MIGLYOL }{ }^{\circledR} 812 \\
\quad \text { Surfactant: PS60 }\end{array}$ & $\begin{array}{l}152.4 \pm 25.2 \\
\text { to } \\
194.5 \pm 18.3\end{array}$ & $\begin{array}{l}-24.1 \pm 9.7 \\
\text { to } \\
-34.6 \pm 6.7\end{array}$ & $\begin{array}{l}64.2 \pm 9.7 \\
\text { to } \\
88.5 \pm 5.1\end{array}$ & n.t. & (Neves et al., 2013) \\
\hline & $\begin{array}{l}\text { Nanoemulsions } \\
\text { (SDEDDS) }\end{array}$ & $\begin{array}{c}\text { Inner oil phase: PG; } \\
\text { Outer oil phase: EPSO; } \\
\text { Hydrophobic surfactant: PGPR; } \\
\text { Hydrophilic surfactant: PS60; } \\
\text { TRANSCUTOL }{ }^{\circledast}\end{array}$ & $12950 \pm 113$ & n.i. & 98 & In vitro: porcine ear skin & (Hu et al., 2016) \\
\hline $\begin{array}{l}\text { Resveratrol + } \\
\text { Curcumin }\end{array}$ & $\begin{array}{l}\text { Nanoemulsion } \\
\text { (SNEDDS) }\end{array}$ & $\begin{array}{c}\text { Oil phase: } \text { LABRAFAC }^{\circledR} \text { or } \\
\text { LABRAFAC }{ }^{\oplus G} \\
\text { Surfactant: } \text { PS80 or } \\
\text { CREMOPHOR }^{\oplus} \text { RH40; Hyaluronic } \\
\text { acid }\end{array}$ & $\begin{array}{c}115.2 \pm 0.1 \\
\text { to } \\
229.5 \pm 13.7\end{array}$ & $\begin{array}{l}-23.9 \pm 1.7 \\
\text { to } \\
-18.6 \pm 0.1\end{array}$ & n.i. & $\begin{array}{c}\text { In vivo: rats } \\
\text { Intranasal administration } \\
\text { In vitro: rats' brain tissue } \\
\text { Ex vivo: Sheep nasal } \\
\text { mucosa }\end{array}$ & (Nasr, 2016) \\
\hline Rosmarinic acid & SLN & $\begin{array}{l}\text { Solid Lipid: WITEPSOL }{ }^{\circledR} \mathrm{H} 15 \\
\quad \text { Surfactant: PS80 }\end{array}$ & $\begin{array}{l}372 \pm 80 \\
\text { to } \\
1400 \pm 31\end{array}$ & $\begin{array}{l}-38.0 \pm 2.9 \\
\text { to } \\
-39.4 \pm 3.1\end{array}$ & $\begin{array}{l}99.8 \\
\text { to } \\
99.9\end{array}$ & n.t. & (Campos et al., 2014) \\
\hline
\end{tabular}


Table 3 (continued)

\begin{tabular}{|c|c|c|c|c|c|c|c|}
\hline \multirow[b]{2}{*}{ Compounds } & \multirow[b]{2}{*}{ Nanocarriers } & \multirow[b]{2}{*}{ Composition } & \multicolumn{3}{|c|}{ Characterization of the nanocarriers } & \multirow{2}{*}{$\begin{array}{c}\text { Experimental model } \\
\text { (route of administration) }\end{array}$} & \multirow[b]{2}{*}{ Ref. } \\
\hline & & & Size (nm) & $\begin{array}{c}\text { Zeta } \\
\text { potential } \\
(\mathrm{mV})\end{array}$ & EE (\%) & & \\
\hline Safranal & $\begin{array}{l}\text { Nanoemulsion } \\
\text { (SNEDDS) }\end{array}$ & $\begin{array}{c}\text { Oil phase: Safranal } \\
\text { Surfactant: PS20, LABRASOL } \\
\text { CREMOPHOR } \\
\text { CoL } \\
\text { Co-surfactant: TRANSCUTOL } \\
\text { Surface coating: CHS }\end{array}$ & $\begin{array}{c}27.1 \pm 6.3 \\
\text { to } \\
89.6 \pm 9.1\end{array}$ & $\begin{array}{l}-11.3 \pm 0.8 \\
\text { to } \\
-29.4 \pm 1.1\end{array}$ & $\begin{array}{l}97.4 \pm 0.8 \\
\text { to } \\
99.9 \pm 0.3\end{array}$ & $\begin{array}{c}\text { In vivo : rats } \\
\text { i.v. administration } \\
\text { Intranasal administration } \\
\text { In vitro: rats' brain tissue }\end{array}$ & (Ahmad et al., 2017) \\
\hline Salvianolic acid B & Liposomes & PC, CHOL, PEG2000 & $140.0 \pm 6.5$ & $-7.9 \pm 0.5$ & $22.7 \pm 0.9$ & $\begin{array}{c}\text { In vivo: rats } \\
\text { i.p. administration } \\
\text { In vitro: rats' brain tissue }\end{array}$ & (Isacchi et al., 2011) \\
\hline Sasanquasaponin & Liposomes & PC, CHOL, PS80, Phaeophorbide & $172.2 \pm 9.7$ & $-60.2 \pm 1.5$ & $84.2 \pm 5.3$ & $\begin{array}{l}\text { In vivo : mice } \\
\text { i.v. administration }\end{array}$ & (Ye et al., 2014) \\
\hline Schisantherin A & $\begin{array}{l}\text { Nanoemulsion } \\
\text { (SNEDDS) }\end{array}$ & $\begin{array}{l}\text { Oil phase: Soy bean oil } \\
\text { Surfactant: PEG15H }\end{array}$ & n.i. & n.i. & n.i. & $\begin{array}{l}\text { In vivo : rats } \\
\text { Oral administration }\end{array}$ & (Sa et al., 2015a) \\
\hline Sesamol & SLN & $\begin{array}{l}\text { Solid Lipid: COMPRITOL }{ }^{\circledR 8} 88 \text { ATO } \\
\text { Surfactant: SPC; PS80 }\end{array}$ & 40 to 70 & n.i. & $75.9 \pm 2.91$ & $\begin{array}{c}\text { In vivo: rats } \\
\text { Oral administration } \\
\text { In vitro: rats' brain tissue }\end{array}$ & (Sachdeva et al., 2015) \\
\hline \multirow[t]{2}{*}{ Thymoquinone } & $\begin{array}{l}\text { Nanoemulsion } \\
\text { (SNEDDS) }\end{array}$ & $\begin{array}{c}\text { Oil phase: Oleic acid } \\
\text { Surfactant: PS20, LABRASOL } \\
\text { CREMOPHOR } \\
\text { CRL }^{\oplus} \\
\text { Co-surfactant: TRANSCUTOL }{ }^{\otimes} \\
\text { Surface coating: CHS }\end{array}$ & $\begin{array}{c}94.8 \pm 6.6 \\
\text { to } \\
139.3 \pm 22.1\end{array}$ & $\begin{array}{l}-13.5 \pm 1.0 \\
\text { to } \\
-31.1 \pm 2.6\end{array}$ & $\begin{array}{l}97.4 \pm 0.8 \\
\text { to } \\
99.9 \pm 0.3\end{array}$ & $\begin{array}{c}\text { In vivo: rats } \\
\text { i.v. administration } \\
\text { Intranasal administration } \\
\text { Ex vivo: Goat nasal mucosa }\end{array}$ & (Ahmad et al., 2016) \\
\hline & SLN & $\begin{array}{l}\text { Solid Lipid: SA } \\
\text { Surfactants: PC, Sodium } \\
\text { taurocholate }\end{array}$ & $172.1 \pm 7.4$ & n.i. & n.i. & $\begin{array}{c}\text { In vivo: rats } \\
\text { Oral administration } \\
\text { In vitro: rats' brain tissue }\end{array}$ & $\begin{array}{l}\text { (Ramachandran and } \\
\text { Thangarajan, 2016) }\end{array}$ \\
\hline
\end{tabular}

AD: Alzheimer disease; $\beta$-CD: 2-hydroxypropyl- $\beta$-cyclodextrin; ApoE: Apolipoprotein E; BCEC cells: brain capillary endothelial cells; CA: Citric Acid; CAPMUL ${ }^{\circledR}$ MCM: mono-diglyceride of medium chain fatty acids (mainly caprylic and capric); Caco-2 cells: heterogeneous human epithelial colorectal adenocarcinoma cells; CAPTEX ${ }^{\circledR}$ 500: glyceryl triacetate or triacetin; CHOL: Cholesterol; CHOL-O: Cholesteryl oleate; CHS: Chitosan; CO: Castor oil; COMPRITOL ${ }^{\circledR 888}$ ATO: blend of different esters of behenic acid with glycerol or glyceryl behenate; CREMOPHOR ${ }^{\circledR}$ EL: KOLLIPHOR ${ }^{\circledR}$ EL or Macrogolglycerol ricinoleate or PEG-35 castor oil or Polyoxyl 35 hydrogenated castor; CREMOPHOR ${ }^{\circledR}$ RH40: KOLLIPHOR ${ }^{\circledR}$ RH40 or Macrogolglycerol hydroxystearate or PEG-40 castor oil, Polyoxyl 40 hydrogenated castor oil; DBC cells: Dissociated brain cells; DCP: Dicetylphosphate or dihexadecyl phosphate; DOPE-PEG-N3: 1,2-dioleoyl-sn-glycero-3-phosphoethanolamine grafted with PEG azide; DPPC: 1,2-dihexadecanoyl-sn-glycero-3-phosphocholine or Dipalmitoyl phosphatidylcholine or 1,2-dipalmitoyl-sn-glycero-3-phosphocholine or 1,2-Dipalmitoyl-3sn-phosphatidylcholine or 1,2-Dipalmitoyl-L-lecithin; DPPG: 1,2-dihexadecanoyl-sn-glycero-3-phospho-(1'-rac-glycerol) or 1,2-dipalmitoyl-sn-glycero-3-phospho-(1'rac-glycerol); DPS-CUR: conjugate of phospholipid (1,2- dipalmitoyl-3-(2-(1,7-bis(4-hydroxy-3-methoxyphenyl)-3,5- dioxohept-6-enylthio)ethyl phospho)-sn-glycerol) and curcumin; DSPE-PEG2000: Conjugate of 1,2- distearoyl sn-glycero-3-phosphoethanolamine and polyethylene glycol (2000) monostearate DPS-PEG2000CUR: Conjugated of DSPE-PEG2000 and curcumin; DSPE-PEG2000 maleimide: 1,2- distearoyl sn-glycero-3-phosphoethanolamine-N-(maleimide(polyethylenoglycol)-2000; DSPC: 1,2-distearoyl-sn-glycero-3-phosphocholine or 1,2-dioctadecanoyl-sn-glycero-3-phosphocholine; EGCG: Epigallocatechin gallate; EPC: egg phosphatidylcholine; EPSO: evening primrose seed oil; G62: GELUCIRE $62 / 5^{\circledR}$ i.e. family of lipid-based excipients comprising mono-, di-, and triglycerides and mono- and di-fatty acid esters of PEG, in this case 62 is referring to the nominal melting point of the base and 5 is the hydrophilic-lipophilic balance; GMS: Glycerol monostearate; hAPPsw SH-SY5Y cells: SH-SY5Y cells stably overexpressing the human APP gene (hAPP) bearing the Swedish mutation (causing familial Alzheimer disease); HBLEC cells: Human brain-like endothelial cells obtained by culturing endothelial cells derived from hematopoietic stem cells isolated from umbilical cord blood; hCMEC/D3 cells: Immortalized human brain capillary endothelial cells; HEK cells: cells from Human Embryonic Kidney; HSPC: Hydrogenated soybean phosphatidylcholine or L- $\alpha$-phosphatidylcholine, hydrogenated (Soy) or PHOSPHOLIPON ${ }^{\circledR} 90$ H; HUVEC cells: human umbilical vein endothelial cells; i.c.v administration: intracerebroventricular administration i.p. administration: intraperitonal administration; i.v. administration: intravenous administration; LABRAFAC ${ }^{\circledR}$ : Caprylic/Capric triglyceride; LABRAFAC ${ }^{\circledR}$ PG: Propylene glycol dicaprylocaprate; LABRASOL ${ }^{\circledR}$ : mono-, di- and triglycerides and mainly PEG-8 (MW 400) mono- and diesters of caprylic (C8) and capric (C10) acids also designated as Caprylocaproyl Polyoxyl-8 glycerides or PEG-8 caprylic/capric glycerides; LB-509: $\alpha$-synuclein antibody; Lf: Lactoferrin; mAb: monoclonal antibody; MAN: p-aminophenyl- $\alpha$-D-mannoside; MIGLYOL ${ }^{\oplus} 812$ : caprylic/capric triglycerides; MYRJ ${ }^{\oplus 52:}$ Polyoxyethylene (40) stearate; n.i.: not indicated; n.t.: not tested; OX26: Transferrin antibody; P188: Poloxamer 188 or PLURONIC F68 ${ }^{\circledR}$ or KOLLIPHOR P188 ${ }^{\circledR}$ or LUTROL F68 ; PC: 1,2-diacyl-sn-glycero-3-phosphocholine or Phosphatidylcholine or Diacylphosphatidylcholine or lecithin or EPIKURON ${ }^{\circledR} 200$ or PHOSPHOLIPON ${ }^{\circledR}$ 90 G; PA: Palitic acid; PC12 cells: cells derived from a pheochromocytoma of the rat adrenal medulla; PE: 1,2-diacyl-sn-glycero-3-phosphoethanolamine or phosphatidylethanolamine ; PEG: poly(ethyleneglycol); PEG15H: Macrogol 15 hydroxystearate or Polyoxyl 15 Hydroxystearate or SOLUTOL HS ${ }^{\circledR} 15$ or KOLLIPHOR ${ }^{\circledR}$ HS15 or PEG-660 stearate; PEG100: polyethylene glycol (100) monostearate; PEG400: polyethylene glycol (400) monostearate; PEG660: polyethylene glycol (660) monostearate PEG2000: polyethylene glycol (2000) monostearate; PG: propylene glycol; PGPR: polyglycerol polyricinoleate; PVA: Poly(vinyl alcohol); PRECIROL ${ }^{\circledR} 5$ ATO: Glyceryl palmitostearate; PS20: Polysorbate 20 or TWEEN ${ }^{\circledR} 20$ or polyoxyethylene sorbitan monolaurate; PS60: Polysorbate 60 or TWEEN ${ }^{\circledR} 60$ or polyoxyethylene sorbitan monostearate; PS80: Polysorbate 80 or TWEEN $^{\circledR} 80$ or polyoxyethylene sorbitan monooleate; SA: Stearic acid or octadecanoic acid or nOctadecanoic acid; SH-SY5Y cells or SK-N-SH cells: human neuroblastoma cells; SPC: soybean phosphatidylcholine; TG (16:0): 1,2,3-trihexadecanoyl-sn-glycerol or Tripalmitin or 1,2,3-trihexadecanoyl-glycerol; TG (18:1): Triolein or glyceryl trioleate or 1,2,3-tri-oleoyl-glycerol or 1,2,3-tri-(9Z-octadecenoyl)-glycerol or Glyceryl trioleate; TPGS: D- $\alpha$-tocopheryl polyethylene glycol 1000 succinate; TRANSCUTOL ${ }^{\oplus}$ : Diethylene glycol monoethyl ether or Ethoxydiglycol or Carbitol; Vit E: Vitamin E or Tocopherol; WITEPSOL ${ }^{\circledR}$ H15: composed by a blend of hydrogenated coco-glycerides (C12-C18). 
methods need low energy as the fabrication is dependent on the oil's and surfactant's physicochemical characteristics and on the modulation of interfacial phase transitions. On the other hand, as the name implies, uses devices that take advantage of a very high energy to produce nanoemulsions. These method includes the high-pressure homogenization, which is the most common method used for nanoemulsions production. (Date et al., 2010).

\subsubsection{Self-Nanoemulsifying drug delivery system (SNEDDS)}

SNEDDS are defined as thermodynamically unstable isotropic mixtures constituted by oil, surfactants, and drug, and when these SNEDDS components are introduced into aqueous environment below moderate agitation, they promptly and spontaneously originate $\mathrm{O} / \mathrm{W}$ nanoemulsions (Fig. 4) (Date et al., 2010). These systems have the ability to emulsify nanodroplets of oil in water due to surfactants that are added to the oily phase (a surfactant and sometimes also a co-surfactant is added to improve the drug entrapment and simplify nanoemulsification). The process of self-emulsifying depends on the surfactant's and oil's nature, as well as on the surfactant's concentration and the temperature in which the process occurs [1]. Also, in human body, the digestive motility of the gastrointestinal tract (GIT) provides the mechanical strength neededgett to help the nanoemulsion formation (Date et al., 2010).

In direct contrast with the current nanoemulsions, SNEDDS present many advantages such as: i) drug selective targeting due to GIT specific absorption; ii) controlled delivery profiles; iii) improved stability profiles even in long-term storage and iv) ability to fill SNEDDs into unit dosage forms (Date et al., 2010; Pujara, 2012).

\subsubsection{Self-Double-Emulsifying drug delivery system (SDEDDS)}

SDEDDS are polydispersed systems that promisingly emerged in order to increase the oral bioavailability of numerous drugs that have low water solubility, allowing the transport of compounds in droplets of oil, during its route through the GIT. In other words, these type of emulsions holds great utility in prolonged delivery of drugs once the drug located in most inner phase is forced to partition itself over numerous phases prior to release at the site of action (Chourasia and Khutle, 2015; Pujara, 2012).

SDEDDS arose from the SNEDDS concept with the purpose of increasing drug bioavailability with low permeability and high solubility (Chourasia and Khutle, 2015). To accomplish this intent, SDEDDS are comprised by formulated mixtures of hydrophilic surfactants and water-in-oil emulsions, that resembling SNEEDS, are able to spontaneously emulsify in the mixed aqueous gastrointestinal environment giving rise to water-in-oil-in-water emulsions (W/O/W SDEDDS Fig. 4) (Qi et al., 2011). Non-aqueous SDEDDS can be also produced by formulating mixtures of oil-in-oil and lipophilic stabilizers, that when mixed with hydrophilic surfactants can spontaneously emulsify in oilin-oil-in-water emulsions (O/O/W SDEDDS Fig. 4) (Hu et al., 2016).

\section{Lipid nanocarriers containing natural compounds as delivery systems treating age related neurodegenerative diseases}

Encapsulating natural compounds in lipid NC conjugates the beneficial neuroprotective effects of the phytochemicals (described in Table 1) with the improvement of their pharmacokinetic profile provided by the NC. Furthermore, as previously stated, lipid NC can be made to imitate LDL and be able to interact with LDL receptors, which triggers uptake by the brain's endothelial cells. Table 3 depicts an overview of reported lipid NC containing natural compounds used for their neuroprotective effects and/or prospective treatment of ARND and the following subsections summarize the most relevant achievements of these studies.

\subsection{Improvements in bioavailability and pharmacokinetic profile of natural compounds}

Poor bioavailability is one of major hurdles in therapeutic development, particularly in the area of CNS mainly due to natural compounds' poor solubility and membrane permeability that is limited, metabolism of the gut-wall, and transporter-mediated intestinal secretion. Furthermore, natural compounds present poor pharmacokinetic profiles and a short half-life being rapidly and extensively metabolized and excreted. This implies that large doses of natural compounds are required to reach therapeutically effective plasma concentrations (Huang et al., 2011; Wei et al., 2014) (Blasina et al., 2015). Therefore, enhancing the natural compounds bioavailability and pharmacokinetic profile is urgently needed and lipid NC address this problem.

Liposomes have improved quercetin, baicalin, curcumin and salvianolic acid B bioavailability in animal models (Blasina et al., 2015; Meng et al., 2015; Wei et al., 2014). Quercetin plasma levels measured after the administration of liposomal were $1.2 \pm 0.8 \mathrm{mg} / \mathrm{mL}$ at half an hour and $1.1 \pm 0.7 \mathrm{mg} / \mathrm{mL}$ at one hour in comparison with free quercetin for which the samples of basal plasma showed no natural compound content (Blasina et al., 2015) Six hours after the administration of the liposomal formulation, quercetin was still available (Blasina et al., 2015). Regarding baicalin, liposomes significantly increased its content in the liver to 5.59-fold, the spleen to 2.33-fold, and the lung to 1.25-fold respectively (Wei et al., 2014). Furthermore, the curcumin concentration in the blood after curcumin-loaded liposomes are intravenously administered at $4 \mathrm{~h}$ was $13.03 \mathrm{ng} / \mathrm{mL}$, while the free curcumin concentration in the blood reduced drastically in the first $2 \mathrm{~h}$ and by $4 \mathrm{~h}$ was undetectable. (Meng et al., 2015). Moreover, compared with free curcumin solution, curcumin-loaded liposomes showed 2.55 folds higher area-under-curve (AUC) and a prolonged stay in a body had a multiple of 4.49 greater mean residence time (MRT) and a 1.81 times lesser chances of being eliminated from the body. (Meng et al., 2015). Salvianolic acid B-loaded PEGylated liposomes also displayed a concentration of plasma that was over the 4-fold compared to unloaded compound and consequently the in vivo efficacy of the liposomal loaded compound was also increased (Isacchi et al., 2011).

Ethosomes, were also successful increasing $( \pm$ ) catechin plasma level in comparison to an equivalent aqueous solution. Consequently, ethosomes delivered $( \pm)$ catechin to different regions of the brain (thalamus, hippocampus, and cortex, striatum) with comparatively high concentrations in relation to those that come from the aqueous solution (Huang et al., 2011).

Micelles were used to improve curcumin's bioavailability approximately 10 to 40 -fold in brain tissue and murine plasma (Hagl et al., 2015).

Nanoemulsions significantly increased the bioavailability of schisantherin A to $47.3 \%$. Furthermore, intranasal administration of nanoemulsions increased about 7 and 9-fold the AUC in brain rat tissues for resveratrol and curcumin, respectively (Nasr, 2016). Pharmacokinetic parameters obtained after intranasal and i.v. administration of thymoquinone mucoadhesive nanoemulsion compared with free thymoquinone were significantly improved in brain, lungs and plasma. Additionally, results clearly showed that when administered through intranasal route the developed nanoemulsion was found to be very effective in enhancing brain thymoquinone bioavailability (Ahmad et al., 2016).

Finally, lipid nanoparticles, such as SLN and NLC were also used to improve bioavailability of natural compounds. NLC doubled the oral bioavailability of EGCG in animal models, making it more effective, even at reduced concentrations (Smith et al., 2010). NLC also increased the plasma level and half-life $\left(\mathrm{t}_{1 / 2}\right)$ of baicalein compared to an equivalent aqueous solution and showed a protective effect of the natural compound against chemical instability. The efficiency of brain targeting by baicalein was improved greatly by NLC, especially to the cortex and brain stem (Tsai et al., 2012). Developed SLN and NLC were 
also able to advance quercetin's relative bioavailability by 3.5 -fold and 5.4-fold, respectively in comparison with free compound. The process of absorption by NLC (at $0.48 \mathrm{~h}^{-1}$ ) was quicker compared to SLNs absorption process (at $0.39 \mathrm{~h}^{-1}$ ) and that of free compound $\left(0.37 \mathrm{~h}^{-1}\right.$ ). Furthermore, SLN and NLC managed to delay the quercetin clearance by 3.5 times and 5.8 times, respectively, which pointed to a significant increase in quercetin biological residence when it is administered encapsulated in NC. Plain quercetin was eradicated largely after $6 \mathrm{~h}$ elapsed since administration, while the compound delivered using nanoparticles was retained in fairly large amounts. The NC were also able to enhance quercetin's the brain delivery by a multiple of 5.6 (NLC) and 3.2 (SLN).These findings demonstrated the superiority of NC (SLN and NLC), generally and NLC, specifically to increase quercetin's that is poor and increase the period of residence in the body and consequently increase its brain distribution (Kumar et al., 2016). The bioavailability of curcumin was also significantly increased when it was included in the SLN and its pharmacokinetic parameters improved (32-155 times) as well as its pharmacodynamics parameters (3-4 times). The presence of SLN containing curcumin in brain and plasma pointed to the delivery of the NC across the BBB and gut wall and as effective. Curcumin delivered by these NC into the brain had 30 times more preferential distribution, which ascertained their promising use in the treatment of ARND (Kakkar and Kaur, 2011; Kakkar et al., 2013a, b). SLN of palmitic acid and cholesterol decorated with chitosan were also able to increase AUC and $t_{1 / 2}$ of curcumin and had a higher brain distribution that unloaded curcumin (Ramalingam and Ko, 2015). Also, using SLN was possible to increase the $t_{1 / 2}$ of luteolin of about $2 \mathrm{~h}$. The clearance and distribution of luteolin with SLN were substantially reduced by 2.16 fold and 10.57 fold, respectively. Therefore SLN containing luteolin showed 4.89 times more bioavailability than unloaded luteolin (Dang et al., 2014). Bioavailability of piperine was also enhanced by encapsulation of this natural compound in SLN reaching higher maximum plasma concentrations $(121 \pm 6.78 \mathrm{ng} / \mathrm{g})$ and 2 fold AUC compared to unloaded piperine (51 $\pm 9.34 \mathrm{ng} / \mathrm{g}$ ) (Yusuf et al., 2013). Finally, SLNs prolonged resveratrol release up to $120 \mathrm{~h}$ and the lipid formulation produced an 8-fold increase in oral resveratrol bioavailability in rats. The $t_{1 / 2}$ was found to increase from $2.4 \mathrm{~h}$ to $11.5 \mathrm{~h}$ when resveratrol was loaded in SLNs (Pandita et al., 2014).

The reasons pointed for the success of lipid NC improving oral bioavailability and pharmacokinetic profile of natural compounds are the small size and composition of the NC (Blasina et al., 2015; Wei et al., 2014). The reduced size of the NC increases the lipid surface area and consequently improves: (i) dissolution rate of the natural compounds; (ii) permeability of the natural compounds through biological membrane (iii) and absorption of the natural compounds in the gastrointestinal tract (i.e. bioavailability increase). The NC lipid composition has also a role on GIT absorption. Lipid NC that mimic the physiological micelles (formed in the intestine) will be taken up from gut to blood by the phagocytotic Peyer patch $\mathrm{M}$ cells and will consequently improve the absorption of lipophilic ingredients. Another possibility for the enhanced absorption, is the increased partitioning of natural compounds encapsulated in lipid NC from the GIT to the gut wall, in comparison with the free compounds, which eventually results in passive permeation that is enhanced across this biomembrane.

Specific elements of lipid NC composition were also pointed out as important in enhancing absorption of natural compounds. This is the case of long-chain triglycerides and surfactants (such as polysorbate 80, lecithin or other phospholipids) that promote the lymphatic absorption of the actives from GIT. This prevents the first-pass metabolism and may lead to permeation improvements of natural compounds via the GI membrane, resulting in an increased oral absorption (Sa et al., 2015a; Wei et al., 2014). The bioadhesive character of these components can also rise the NC's and GI membrane's affinity between them. The resultant longer residence time at the absorption site would assist in increasing the natural compounds uptake. The same role of increasing the retention and penetration of SLNs in the GI tract was achieved by decorating the surface of SLNs with N-trimethyl chitosan (Ramalingam and Ko, 2015, 2016).

Moreover, the role of polysorbate 80 (or TWEEN ${ }^{\circledR} 80$ ) in absorption cannot be ignored. The P-glycoprotein efflux system is also fairly inhibited by polysorbate 80 leading to improved delivery of natural compounds. The same role had the surfactant Brij 78 and D- $\alpha$-tocopheryl polyethylene glycol 1000 succinate (TGPS) used to decorate curcumin-loaded SLNs and to inhibit the P-glycoprotein efflux pump with a resultant permeability enhancement of 1.3 times and 1.4 times greater than conventional SLNs in the jejunum (Ji et al., 2016).

Furthermore polysorbate 80 can also give raise to steric hindrance in lipid NC providing protection and resistance to NC elimination from the blood circulation (Hagl et al., 2015; Huang et al., 2011; Wei et al., 2014). In addition, cholesterol in lipid NC is likely to supply better resistance against pancreatic lipase thereby can be used to guard the natural compound from an intestinal medium attack (Blasina et al., 2015; Huang et al., 2011). Gelucires ${ }^{\circledR}$ (a lipid-based excipients family made up of a mixture of glycerides and PEGylated fatty acid esters) and vitamin $\mathrm{E}$ are also described as playing important roles in improving lipid NC resistance and enhancing the pharmacokinetics and brain transport (Tsai et al., 2012).

As a final point, the lipid composition is also important to assure the integrated natural compound's sustained release in a system of delivery which is a crucial property that often correlates with improved efficacy and pharmacokinetics (Huang et al., 2011; Tsai et al., 2012).

\subsection{Administration routes to deliver natural compounds to brain}

The administration routes used to deliver lipid NC containing natural compounds can be classified into two major categories: local NC delivery and systemic administration. Local NC delivery have the advantage of providing effective therapeutic concentrations of bioactives in the brain guaranteeing total bioavailability of the bioactive compound and avoiding all the physiological barriers that bioactives would encounter, including the BBB. However, local NC delivery requires surgeries or invasive methods to place NC in the brain. Moreover additional surgeries may be necessary if the treatment requires multiple doses. Examples of local NC delivery include intracerebroventricular administration (i.c.v) or implantation by stereotaxic administration (a minimally invasive form of surgical intervention which makes use of a three-dimensional coordinate system to place NC). Due to the technical requirements of this type of local administration route only few studies have used it (Lazar et al., 2013; Vedagiri and Thangarajan, 2016).

The majority of lipid based NC loaded with bioactives are administered to CNS systemically, most likely via intravenous (i.v.) injection because of the ease of application and avoidance of the first pass effect (Ahmad et al., 2017, 2016; Blasina et al., 2015; Dhawan et al., 2011; Meng et al., 2015; Rivera et al., 2008; Sarkar and Das, 2006; Tsai et al., 2012; Ye et al., 2014). Other systemic administrations include: oral administration (Dang et al., 2014; Hagl et al., 2015; Huang et al., 2011; Kakkar and Kaur, 2011; Kakkar et al., 2013a, b; Kumar et al., 2016); intraperitoneal administration (i.p) (Galho et al., 2016; Isacchi et al., 2011; Puglia et al., 2012; Wang et al., 2015; Yan et al., 2014; Yusuf et al., 2013; Zhao et al., 2011) and transdermal administration (Hu et al., 2016; Shi et al., 2012).

Intranasal administration has been investigated recently as an alternative to i.v. administration as it is an opportunity to bypass BBB by transferring the NC from the olfactory mucosa directly to the CNS through the olfactory pathway. Therefore this route gives direct access of NC loaded with bioactives to the brain and offers a new means for the long-term non-invasive management of chronic neurological disorders and diseases such as Alzheimer's disease. The benefits that accrue to nasal administration as opposed to oral route are higher bioavailability because of lack of first pass hepatic metabolism and subsequent rapid absorption that result in a shorter period to the start of the effect (Pardeshi and Belgamwar, 2013). In this context, nasal administration 
provides easy brain penetration and a shorter distance to the brain target to NC containing natural bioactives (Ahmad et al., 2017; Nasr, 2016; Sood et al., 2014; Tong-un et al., 2010).

\subsection{Stealth, targeting and triggering strategies to deliver natural compounds} to brain

\subsubsection{Stealth strategies}

In order to prepare long circulating NC, a PEGylation (PEG) coating is needed to give stealth properties to avoid recognition of the NC by the RES system and also to serve BBB targeting ligands that are used in active targeting strategies as an anchor point. PEGylation is a strategy widely used in nanotherapy and ARND nanotherapy is no exception, as additionally it has been documented that PEG coated NC may have increased affinity for the BBB endothelial cells in a brain that is normal (Brasnjevic et al., 2009). Therefore, many authors have used PEG as a stealth strategy in lipid NC containing natural compounds for brain delivery (Isacchi et al., 2011; Meng et al., 2015; Mourtas et al., 2011, 2014; Phachonpai et al., 2010; Sood et al., 2014; Tong-un et al., 2010; Yan et al., 2014).

This was the case of liposomes containing salvianolic acid, where the PEGylation has been associated with an increase of the natural compound plasmatic concentration over 4-fold compared to other formulations. In this study the inclusion of PEG in the liposomes surface may have modified the salvianolic acid's pharmacokinetic profile, its plasma protein binding and/or its metabolism (Isacchi et al., 2011).

\subsubsection{Active targeting strategies}

Surface functionalization with specific ligands is an active targeting strategy that directs lipid based NC to some receptors that are overexpressed in brain capillary endothelial cells. One literature example of this strategy was the functionalization of NLC surface with lactoferrin (Lf) for curcumin brain delivery (Meng et al., 2015). Lf is a naturally occurring cationic glycoprotein that is iron binding and is capable of being moved across BBB through lactoferrin receptors (LfR). Moreover, an increase of LfR in the brain capillary endothelial cells has been found in PD and AD (Meng et al., 2015). In this context, curcumin loaded LfNLC with Lf at high levels had higher uptakes (1.39 times higher) by the brain capillary endothelial cells than naked NLC (Meng et al., 2015). Additionally, FRET studies showed that curcumin was still inside NLC after uptake by brain capillary endothelial cells, showing the carrier's stability travelled across the BBB. Studies of Ex vivo imaging also confirmed that Lf-NLC could permeate BBB effectively and accumulate preferentially in the brain (Meng et al., 2015). In agreement with the higher brain concentration of Lf-NLC, the Lf-NLC's superior efficacy in the control of $\mathrm{AD}$ associated damage was confirmed through histopathological evaluation (Meng et al., 2015).

Another active targeting strategy used for the delivery of curcumine across BBB was the functionalization of liposomal surface with monoclonal antibodies anti-transferrin receptor (OX26 an anti-TfR mAb). This strategy was chosen because TfR are highly expressed in BBB endothelial cells. Besides brain targeting capability, these liposomal NC have also demonstrated capacity to target $A \beta$ deposits in the brain through curcumin-derivative molecules presence on their surface. Therefore, these multifunctional formulations could be used for theranostic (therapy by curcumin neuroprotective effect and diagnostic by curcumin labelling of $A \beta$ deposits) purposes (Mourtas et al., 2014). The same strategy of targeting TfR with OX-26 antibody was used in SLN loaded with resveratrol from grape skin and grape seed extracts (Loureiro et al., 2017). OX-26 functionalization increased uptake of resveratrol loaded SLN by BBB cell model and transcytosis across the BBB cell model when compared with SLN functionalized with another antibody (LB 509) or with non-functionalized SLN (Loureiro et al., 2017).

Due to mannose receptors being present in the brain, mannosylated liposomes containing quercetin were also used to attempt carrying out drug delivery that was site specific to cerebral tissue (Sarkar and Das, 2006). The authors of this formulation concluded that the administration of non-encapsulated quercetin in rats had no significant antioxidant effect and thus no significant brain protection in aged or young rat brain (Sarkar and Das, 2006). Contrastingly, a single injection of the same amount of quercetin encapsulated in mannose-grafted liposomes decreased and got the oxidation products to near normal levels. This indicated that, treatment of quercetin encapsulated in mannosylated liposomes conferred protection against oxidation and cerebral edema (Sarkar and Das, 2006).

Another active targeting strategy widely used for brain delivery of natural compounds was coating the lipid NC surface with polysorbate (Wei et al., 2014). Polysorbate has been described as able to facilitate the functionalization of NC surface with LDL receptor ligands (ApoE and/or serum ApoB). Consequently, the NC are targeted towards the LDL receptor (low-density lipoprotein receptors (LDLR)) on the capillary endothelial cells of the brain and are internalized, mimicking natural lipoproteins' transcytosis (Georgieva et al., 2014). Using the same strategy, ApoE functionalized SLN improved resveratrol permeability (1.8-fold higher) into human brain capillary endothelial cells when compared to non-functionalized NC (Neves et al., 2016).

Furthermore, polysorbate as well as other detergents like sodium dodecyl sulfate (SDS), or other solvents like ethanol and DMSO can rise BBB vascular endothelium's permeability. The BBB permeability enhancement is possibly due to the detergent's/solvent's destabilizing effect of a membrane leading to BBB disruption (Brasnjevic et al., 2009). The same BBB permeability enhancement strategy is used in lipid NC containing high quantities of ethanol (ethosomes), for brain delivery of natural compounds (Huang et al., 2011; Shi et al., 2012).

\subsubsection{Passive targeting strategies}

In several neurodegenerative and brain injury diseases non-specific pathologic changes like fenestrations formation may occur. It has been further reported that in the $\mathrm{BBB}$, the fenestrations formation that are $100 \mathrm{~nm}$ in size can allow for molecular aggregates like liposomes to pass, which ordinarily cannot reach the brain. It was also reported that to cross the BBB, the lipophilic vesicles may use transcellular diffusion (Kakkar and Kaur, 2011). In addition, the overexpression of the LDLR in brain capillary endothelial cells gives the benefit of targeting the brain for lipid based NC. Based on these assumptions, lipid NC with a controlled size are reported as able to reach brain tissues even without additional surface functionalization. These passive targeting strategies have indeed been observed for the uptake of SLN containing curcumin by brain endothelial cells (Kakkar et al., 2013b). SLN are reported as being taken up readily by the brain because of their lipid nature and their nanometer size which facilitates their passage across the BBB, thus being suitable for passively target the brain (Sachdeva et al., 2015).

Additional passive targeting strategies include the positively charged NC that can make use of the AMT mechanism of adsorption to the negatively charged surface of brain endothelial cells and transport across BBB. Furthermore, NC covered by positively charged mucoadhesive polymers, prolong the contact time for bioactives in the nasal cavity whereby it provides an opportunity for tight junctions to open hence increasing permeation and sustained bioactive delivery to brain. Chitosan is an example of positively charged mucoadhesive polymer used as coating for nanoemulsions containing curcumin, safranal or thymoquinone for nasal administration (Ahmad et al., 2017, 2016; Sood et al., 2014). Besides mucoadhesive polymers, surfactants and other formulation components like cholesterol also favor the opening of tight epithelial junction in nasal membrane, increasing bioactives' penetration. Accordingly, nasal administration of liposomes containing quercetin has been developed as a possible protection strategy against neurodegeneration and these systems have shown beneficial effects with very low dose of natural compound (Sood et al., 2014). The same strategy of nasal administration and BBB bypass was used by the combination of CREMOPHOR ${ }^{\circ}$ RH 40 (known for its 
enhancement of cell membrane fluidity and thus used as permeation enhancer) and hyaluronic acid (reported to improve the mucosal absorption of drugs) to increase the nose to brain transport of curcumin and resveratrol through the olfactory pathway (Nasr, 2016). This composition was reported as able to reduce mucociliary clearance and increase the persistence of the formulation containing natural compounds in the nasal cavity without causing any tissue damage or epithelial and ciliotoxicity (Nasr, 2016).

\subsubsection{Triggering strategies}

To attain NC controlled release, these can be modified in the structure with triggering strategies such as $\mathrm{pH}$ response, photo response, bioenzymatic response, and thermal response. A recent study, using photo responsive liposomes loaded with sasanquasaponin has used this triggering strategy to control the natural compound release by infrared light that has deep penetration and low tissue damage. The photo responsive liposome was composed of a mixture of lecithin and a photosensitive agent (phaeophorbide) that could be activated by a laser light of $670 \mathrm{~nm}$. Upon excitation, this photosensitive agent is a singlet oxygen or oxygen radicals' generator that damage the phosphodiester bonds in the NC lecithin, this way releasing sasanquasaponin. Additionally, to the controlled release of the natural compound, the advantages of this formulation are the fact that the irradiation and release could be done in nasal cavity, provoking little harm (Ye et al., 2014).

\subsection{Biodegradation/bioelimination of lipid nanocarriers from the brain}

The biological fate of NC is still a research topic that requires more attention. Some NC are not readily eliminated by clearance mechanisms, and can cause brain bioaccumulation prompting additional cytotoxicity (Medina et al., 2007). Although several types of NC composed by inorganic nanomaterials, were more thoroughly studied for their potential toxicity (Costa et al., 2016; Hu and Gao, 2010), fewer studies were concerned with the lipid NC, as these are biodegradable, and for that reason considered to not accumulate in the body and regarded as possibly risk-free (Medina et al., 2007; Qi et al., 2017). Other arguments that hinder further investigation of the biological fate of lipid NC are based, for instance, in the fact that lipid-based parenteral nutrition, has been clinically used since the early 1960s. Furthermore all the lipid components of lipid NC for human use are generally recognized as safe (GRAS) (Blasi et al., 2007). Nevertheless, the effects of NC, even of the ones considered biodegradable, on BBB dysfunction and associated consequences are still not well identified. Thus novel studies dealing with the neurotoxic effects of several classes of NC on the CNS function are compulsory. This is even more important considering that the specific mechanisms through which the NC or its degradation products may exert their toxic effects remain largely unknown and no guidelines are presently available to quantify these effects (Hu and Gao, 2010; Sharma, 2007).

From a general standpoint it is true that lipid NC can be considered as having higher acceptability than other NC made of polymeric or inorganic materials. However it is also important to emphasize that besides the tolerability of the lipid constituents, other components are often used in the formulation of lipid NC. For instance, SLN and NLC include surfactants in their composition, and its tolerability and influence in biodegradation must be considered as well (Blasi et al., 2007). For instance, the biodegradation has shown to be slower when triglycerides with long chain fatty acids and/or sterically hindering surfactants like Poloxamer 407 or Poloxamine 908 were part of SLN composition. Contrastingly, a faster degradation was achieved when the lipid nanoparticles composition includes short chain fatty acid triglycerides and/or degradation promoting surfactants like bile salts (e.g. cholic acid sodium salt) (Olbrich et al., 2002). Furthermore, it has also to be considered the influence of particle size on biodegradation rate as this has shown to be different for certain surfactants (Olbrich et al.,
2002). All in all it may be assumed that several factors affect the biodegradation of lipid based NC whose rate depends on several factors such as the lipid composition, particle size, surface decoration, presence of lipases (body enzymes capable of lipolysis) (Qi et al., 2017).

Additionally, the degradation products resultant from lipolysis should not be seen as innocuous risk-free metabolites, especially considering that brain is the organ with highest concentration of lipids next to adipose tissue, demonstrating that lipids metabolism have important role in the CNS (Adibhatla and Hatcher, 2008). Important proofs of the lipids role in brain were given by more and more studies that demonstrated that neurons located at specific regions of the brain (e.g. hypothalamus, hippocampus or striatum) are sensitive to free fatty acids which presence are able of changing their nervous activity (CrucianiGuglielmacci and Magnan, 2017). Therefore, it is not difficult to assume that a local hydrolysis of lipid NC may also participate to brain lipid sensing due to the free fatty acids produced that could then act in the sensitive neurons. Furthermore, phospholipids can also result from degradation of lipid NC and exert their role as precursors of inflammation mediators such as arachidonic acid a substrate of phospholipase A2 (Adibhatla and Hatcher, 2008). Moreover, the products of degradation of lipid NC, free fatty acids and phospholipids, can both suffer another pathway of degradation: lipid oxidation that occurs in astrocytes, resulting in elevated ketone levels in the brain (Bruce et al., 2017; Foradada et al., 2000). The brain is especially vulnerable to this oxidation products as it has a high oxygen consumption rate and reduced antioxidant enzymes ( $\mathrm{Hu}$ and Gao, 2010), and hence the oxidative stress products resultant from lipid NC degradation can lead to inflammation and neuron injury.

The lipid role in brain and in neurodegenerative diseases is also highlighted by the fact that lipid rafts (lipid domains enriched in cholesterol, sphingolipids and saturated fatty acids) disruption is an early marker for diagnosis of the disease (Wang and Eckel, 2014). It has been stated that lipid rafts are affected by lipoproteins metabolism which may be linked to atherosclerosis or neurodegenerative diseases (Wang and Eckel, 2014). Considering that lipid NC use the same pathway (e.g. LDL receptors) as lipoproteins to be transported to the brain (Alam et al., 2010) it is tempting to suggest that, like lipoproteins, lipid NC degradation products may also modulate lipid raft signal platforms.

For all the exposed, lipid NC biodegradation and lipid byproducts of such degradation should be carefully analyzed as may be involved in lipid rafts impairment, inflammation processes or neuron modulation.

Besides biodegradability studies, the lipid composition should not provide a guarantee per se of biocompatibility, and their use in brain diseases should be accompanied with more detailed studies. For example, concerning liposomes, several studies provide evidences of hypersensitivity reactions as these NC can activate complement system (CS), and larger liposomes (200 $\mathrm{nm}$ or above) appeared to be stronger CS activators than their smaller counterpart of identical lipid composition and equivalent total surface area (Gregori et al., 2015). Since all the studies refer the CS activation in blood, further studies on liposomal biocompatibility should be conducted in CNS to provide further awareness on the threats associated with this promising targeting systems.

\section{Conclusions and future perspectives}

Considering the overlapping pathways thought to be triggered in the onset of ARND, one proposed option is that treatments can be founded on natural compounds that ally their safe use to their multiple beneficial neuroprotective effects though quantitatively small when alone, are synergistic, especially over the time duration. The potential of nanotechnology makes it possible for it to be utilized in facilitating delivery of natural compounds across the $\mathrm{BBB}$, to help the neurons functional regeneration and improve the neuroprotective strategies. The encapsulation of natural compounds in NC protects them from degradation and improves their half-life in circulation. 
Functionalization with ligands that target proper receptors on the BBB can concentrate NC loaded with natural compounds within BBB cells or promote their entrance in the brain. Thus, nanoencapsulation development strategies to improve the bioavailability and increase the absorption of natural compounds is of uttermost significance as an ARND therapeutic or prophylactic approach. Generally, the studies analyzed in this discussion showed that lipid NC are capable of enhancing oral bioavailability and/or pharmacokinetic profile of natural compounds being also capable of delivering these compounds to brain. Alternatively, intranasal injections facilitate the direct delivery of natural compounds into the brain bypassing the BBB.

However, many issues remain to be considered before we can benefit from suitable nanotechnology-based delivery systems that could lead to improved ARND therapeutic and prophylactic outcomes. First of all, most studies lack of a thorough characterization of the lipid NC developed, or provide an incomplete or non-systematic methodology of formulation development. Indeed, formulation development is often made in a "trial and error" based method and most of the studies do not consider the influence of NC composition in: (i) natural compounds encapsulation and loading efficiency; (ii) NC size, surface charge potential; (iii) ability of NC to completely release the entrapped bioactives; and ultimately (iv) NC efficiency to deliver natural compounds to brain where their effects are evaluated. For instance, despite the recognized benefit of a positively charged surface to be able to cross BBB using AMT mechanisms, the majority of the NC developed displayed a negatively charged surface. Moreover, despite most authors refer the size of the $\mathrm{NC}$ as an important requirement to cross $\mathrm{BBB}$, this is not a concern for many of the formulations developed.

Besides this, caution is needed because a successful transportation of a lipid NC that has natural compounds across in vitro BBB simulated model is not predictive of its therapeutic effect fully, due to the fact that after the bioactive penetrates across the BBB it is equally essential to analyze if its biological activity is retained or not. On the other hand, In vivo models provide evidences on the improvement of natural compounds benefits upon its encapsulation in lipid NC, however studies were mainly examined by acute or short-term animal models after short duration neurotoxic insults. This seems an inappropriate timing as the long latency period of ARND adds another dimension in its complexity wherein the principal mechanisms change with the time course and progression of the diseases. Therefore, to make most out of the potential of lipid NC as delivery systems for natural compounds across BBB, longer term in vivo clinical trials and pharmacokinetic studies are required to affirm the capacity of these NC to enhance the efficacy and bioavailability of natural compounds.

Another issue that requires consideration is the safety of NC that is developed. The toxicity and safety features of NC are significant considerations that require seriousness, need to be understood well and resolved before the implementation of these formulations for clinical use for ARND treatment. In fact, despite being made of biocompatible constituents, the biological fate of lipid NC can be determined not only by their composition but also by their shape, size that ultimately determine their biological interactions. Thus, long term toxicity and pharmacodynamic implications of the off-target actions of these lipid NC should be studied as it can show differential effects on ARND pathology.

Looking back at so many open issues make us think - are lipid NC loaded with natural compounds potential new therapies for ARND? Yes, definitely. Classical therapeutic approaches for AD and other ARND are limited to a single target and have produced disappointing results in clinical trials. There is no doubt of the beneficial role of natural compounds in several targets of ARND pathology. The improvement of safety data knowledge and the optimization of nanoencapsulated formulations of natural compounds are the succeeding mandatory steps, as well as finding specific targeting strategies of diseased areas in the brain and reducing adverse effects to healthy cells. This review and observations of this critical perspective offer several key points that can help identify potentially problematic research approaches and/or interpretation of outcomes in publications, as increasing our comprehension about these diseases challenges will contribute to the development of novel potential therapeutic strategies.

\section{Acknowledgements}

This work was supported by the Portuguese Foundation for Science and Technology (FCT) in the framework of the Strategic Funding UID/ FIS/04650/2013. Marlene Lúcio acknowledges the exploratory project funded by FCT with the reference IF/00498/2012. Telma Soares acknowledges COMPETE 2020 "Programa Operacional Competitividade e internacionalização". The authors thank Cláudia Candeias, for debating the contents of this review.

\section{References}

Adibhatla, R.M., Hatcher, J.F., 2008. Altered lipid metabolism in brain injury and disorders. Subcell. Biochem. 49, 241-268.

Ahmad, N., Ahmad, R., Alam, M.A., Samim, M., Iqbal, Z., Ahmad, F.J., 2016. Quantification and evaluation of thymoquinone loaded mucoadhesive nanoemulsion for treatment of cerebral ischemia. Int. J. Biol. Macromol. 88, 320-332.

Ahmad, N., Ahmad, R., Abbas Naqvi, A., Ashafaq, M., Alam, M.A., Ahmad, F.J., AlGhamdi, M.S., 2017. The effect of safranal loaded mucoadhesive nanoemulsion on oxidative stress markers in cerebral ischemia. Artif. Cells Nanomed. Biotechnol. 45, $775-787$.

Alam, M.I., Beg, S., Samad, A., Baboota, S., Kohli, K., Ali, J., Ahuja, A., Akbar, M., 2010. Strategy for effective brain drug delivery. Eur. J. Pharm. Sci. 40, 385-403.

Albarracin, S.L., Stab, B., Casas, Z., Sutachan, J.J., Samudio, I., Gonzalez, J., Gonzalo, L., Capani, F., Morales, L., Barreto, G.E., 2012. Effects of natural antioxidants in neurodegenerative disease. Nutr. Neurosci. 15, 1-9.

Allen, T.M., Cullis, P.R., 2013. Liposomal drug delivery systems: from concept to clinical applications. Adv. Drug Del. Rev. 65, 36-48.

Aquilano, K., Baldelli, S., Rotilio, G., Ciriolo, M.R., 2008. Role of nitric oxide synthases in Parkinson's disease: a review on the antioxidant and anti-inflammatory activity of polyphenols. Neurochem. Res. 33, 2416-2426.

Ballabh, P., Braun, A., Nedergaard, M., 2004. The blood-brain barrier: an overview. Neurobiol. Dis. 16, 1-13.

Barras, A., Mezzetti, A., Richard, A., Lazzaroni, S., Roux, S., Melnyk, P., Betbeder, D., Monfilliette-Dupont, N., 2009. Formulation and characterization of polyphenolloaded lipid nanocapsules. Int. J. Pharm. 379, 270-277.

Benilova, I., Karran, E., De Strooper, B., 2012. The toxic A $\beta$ oligomer and Alzheimer's disease: an emperor in need of clothes. Nat. Neurosci. 15, 349-357.

Blasi, P., Schoubben, A., Giovagnoli, S., Rossi, C., Ricci, M., 2007. Solid lipid nanoparticles to improve brain drug delivery. Nanotechnologies for the Life Sciences. Wiley-VCH Verlag GmbH \& Co. KGaA, pp. 365-393.

Blasina, F., Vaamonde, L., Silvera, F., Tedesco, A.C., Dajas, F., 2015. Intravenous nanosomes of quercetin improve brain function and hemodynamic instability after severe hypoxia in newborn piglets. Neurochem. Int. 89, 149-156.

Brasnjevic, I., Steinbusch, H.W., Schmitz, C., Martinez-Martinez, P., 2009. Delivery of peptide and protein drugs over the blood-brain barrier. Prog. Neurobiol. 87, 212-251.

Bruce, K.D., Zsombok, A., Eckel, R.H., 2017. Lipid processing in the brain: a key regulator of systemic metabolism. Front. Endocrinol. (Lausanne) 8.

Campos, D.A., Madureira, A.R., Gomes, A.M., Sarmento, B., Pintado, M.M., 2014. Optimization of the production of solid Witepsol nanoparticles loaded with rosmarinic acid. Colloids Surfaces. B Biointerfaces 115, 109-117.

Cardoso, F.L., Brites, D., Brito, M.A., 2010. Looking at the blood-brain barrier: molecular anatomy and possible investigation approaches. Brain Res. Rev. 64, 328-363.

Chen, Y., Liu, L., 2012. Modern methods for delivery of drugs across the blood-brain barrier. Adv. Drug Del. Rev. 64, 640-665.

Chen, X., Guo, C., Kong, J., 2012. Oxidative stress in neurodegenerative diseases. Neural Regen. Res. 7, 376-385.

Chiueh, C.C., Andoh, T., Lai, A.R., Lai, E., Krishna, G., 2000. Neuroprotective strategies in parkinson's disease: protection against progressive nigral damage induced by free radicals. Neurotox. Res. 2, 293-310.

Chourasia, K.J., Khutle, N.M., 2015. Self double emulsifying drug delivery system: a comprehensive review. World J. Pharm. Pharm. Sci. 4, 433-447.

Costa, P.M., Bourgognon, M., Wang, J.T.W., Al-Jamal, K.T., 2016. Functionalised carbon nanotubes: from intracellular uptake and cell-related toxicity to systemic brain delivery. J. Control. Release 241, 200-219.

Cruciani-Guglielmacci, C., Magnan, C., 2017. Brain lipoprotein lipase as a regulator of energy balance. Biochimie 143, 51-55.

Daglia, M., Di Lorenzo, A., Nabavi, S.F., Talas, Z.S., Nabavi, S.M., 2014. Polyphenols: well beyond the antioxidant capacity: gallic acid and related compounds as neuroprotective agents: you are what you eat!. Curr. Pharm. Biotechnol. 15, 362-372.

Dang, H., Meng, M.H.W., Zhao, H., Iqbal, J., Dai, R., Deng, Y., Lv, F., 2014. Luteolinloaded solid lipid nanoparticles synthesis, characterization, \& improvement of bioavailability, pharmacokinetics in vitro and vivo studies. J. Nanopart Res. 16, 1-10.

Date, A.A., Desai, N., Dixit, R., Nagarsenker, M., 2010. Self-nanoemulsifying drug 
delivery systems: formulation insights, applications and advances. Nanomedicine 5, 1595-1616.

Devi, K.P., 2014. Botanics : a potential source of new therapies for Alzheimer's disease? Botanics 11-26.

Dhawan, S., Kapil, R., Singh, B., 2011. Formulation development and systematic optimization of solid lipid nanoparticles of quercetin for improved brain delivery. J. Pharm. Pharmacol. 63, 342-351.

Durrenberger, Pl.F., Fernando, F.S., Kashefi, S.N., Bonnert, T.P., Seilhean, D., Oumesmar, B.N., Schmitt, A., Gebicke-Haerter, P.J., Falkai, P., Grünblatt, E., Palkovits, M. Arzberger, T., Kretzschmar, H., Dexter, D.T., Reynolds, R., 2015. Common mechanisms in neurodegeneration and neuroinflammation: a BrainNet Europe gene expression microarray study. J. Neural Transm. 122, 1055-1068.

Eikelenboom, P., Veerhuis, R., Scheper, W., Rozemuller, A.J., van Gool, W.A., Hoozemans, J.J., 2006. The significance of neuroinflammation in understanding Alzheimer's disease. J. Neural Transm. 113, 1685-1695.

Etheridge, M.L., Campbell, S.A., Erdman, A.G., Haynes, C.L., Wolf, S.M., McCullough, J., 2013. The big picture on nanomedicine: the state of investigational and approved nanomedicine products. Nanomed. Nanotechnol. Biol. Med. 9, 1-14.

EUJPND, 2012. European Joint Programme in Neurodegenerative Disease Research Strategy: Tackling the Challenge of Alzheimer'S and Other Neurodegenerative DiseaseS in Europe. UK Medical Research Council.

Fischer, R., Maier, O., 2015. Interrelation of oxidative stress and inflammation in neurodegenerative disease: role of TNF. Oxid. Med. Cell. Longev. 2015, 1-18.

Foradada, M., Pujol, M.D., Bermúdez, J., Estelrich, J., 2000. Chemical degradation of liposomes by serum components detected by NMR. Chem. Phys. Lipids 104, 133-148.

Fulda, S., Gorman, A.M., Hori, O., Samali, A., 2010. Cellular stress responses: cell survival and cell death. Int. J. Cell. Biol. 1-23.

Gaillard, P.J., Visser, C.C., Appeldoorn, C.C.M., Rip, J., 2012. Enhanced brain drug delivery: safely crossing the blood-brain barrier. Drug. Discov. Today Technol. 9, e155-e160.

Galho, A.R., Cordeiro, M.F., Ribeiro, S.A., Marques, M.S., Antunes, M.F., Luz, D.C. Hadrich, G., Muccillo-Baisch, A.L., Barros, D.M., Lima, J.V., Dora, C.L., Horn, A.P., 2016. Protective role of free and quercetin-loaded nanoemulsion against damage induced by intracerebral haemorrhage in rats. Nanotechnology 27, 175101.

Georgieva, J., Hoekstra, D., Zuhorn, I., 2014. Smuggling drugs into the brain: an overview of ligands targeting transcytosis for drug delivery across the blood-brain barrier. Pharmaceutics 6, 557-583.

Gholamnezhad, Z., Havakhah, S., Boskabady, M.H., 2016. Preclinical and clinical effects of Nigella sativa and its constituent, thymoquinone: a review. J. Ethnopharmacol. 190, 372-386.

Giacoppo, S., Galuppo, M., Montaut, S., Iori, R., Rollin, P., Bramanti, P., Mazzon, E., 2015. An overview on neuroprotective effects of isothiocyanates for the treatment of neurodegenerative diseases. Fitoterapia 106, 12-21.

Godin, B., Touitou, E., 2003. Ethosomes: new prospects in transdermal delivery. Crit. Rev. Ther. Drug Carr. Syst. 20, 63-102.

Gregori, M., Masserini, M., Mancini, S., 2015. Nanomedicine for the treatment of Alzheimer's disease. Nanomedicine 10, 1203-1218.

Griffin, W.S.T., 2006. Inflammation and neurodegenerative diseases. Am. J. Clin. Nutr. 83,470 - $-474 S$.

Haass, C., Selkoe, D.J., 2007. Soluble protein oligomers in neurodegeneration: lessons from the Alzheimer's amyloid $\beta$ peptide. Nat. Rev. Mol. Cell. Biol. 8, 101-112.

Hagl, S., Kocher, A., Schiborr, C., Kolesova, N., Frank, J., Eckert, G.P., 2015. Curcumin micelles improve mitochondrial function in neuronal PC12 cells and brains of NMRI mice - impact on bioavailability. Neurochem. Int. 89, 234-242.

Hu, Y.-L., Gao, J.-Q., 2010. Potential neurotoxicity of nanoparticles. Int. J. Pharm. 394, 115-121.

Hu, C., Wang, Q., Ma, C., Xia, Q., 2016. Non-aqueous self-double-emulsifying drug delivery system: a new approach to enhance resveratrol solubility for effective transdermal delivery. Colloids Surf. Physicochem. Eng. Aspects 489, 360-369.

Huang, Y.B., Tsai, M.J., Wu, P.C., Tsai, Y.H., Wu, Y.H., Fang, J.Y., 2011. Elastic liposomes as carriers for oral delivery and the brain distribution of (+)-catechin. J. Drug Target 19, 709-718.

Isacchi, B., Fabbri, V., Galeotti, N., Bergonzi, M.C., Karioti, A., Ghelardini, C., Vannucchi, M.G., Bilia, A.R., 2011. Salvianolic acid B and its liposomal formulations: anti-hyperalgesic activity in the treatment of neuropathic pain. Eur. J. Pharm. Sci. 44, 552-558.

Jain, K.K., 2006. Role of nanotechnology in developing new therapies for diseases of the nervous system. Nanomedicine 1, 9-12.

Ji, H., Tang, J., Li, M., Ren, J., Zheng, N., Wu, L., 2016. Curcumin-loaded solid lipid nanoparticles with Brij78 and TPGS improved in vivo oral bioavailability and in situ intestinal absorption of curcumin. Drug Deliv. 23, 459-470.

Jo, D.H., Kim, J.H., Lee, T.G., Kim, J.H., 2015. Size, surface charge, and shape determine therapeutic effects of nanoparticles on brain and retinal diseases. Nanomed. Nanotechnol. Biol. Med. 11, 1603-1611.

Jong, W.H.D., Borm, P.J.A., 2008. Drug delivery and nanoparticles: applications and hazards. Int. J. Nanomed. 3, 133-149.

Kabanov, A.V., Gendelman, H.E., 2007. Nanomedicine in the diagnosis and therapy of neurodegenerative disorders. Prog. Polym. Sci. 32, 1054-1082.

Kakkar, V., Kaur, I.P., 2011. Evaluating potential of curcumin loaded solid lipid nanoparticles in aluminium induced behavioural, biochemical and histopathological alterations in mice brain. Food Chem. Toxicol. 49, 2906-2913.

Kakkar, V., Mishra, A.K., Chuttani, K., Kaur, I.P., 2013a. Proof of concept studies to confirm the delivery of curcumin loaded solid lipid nanoparticles (C-SLNs) to brain. Int. J. Pharm. 448, 354-359.

Kakkar, V., Muppu, S.K., Chopra, K., Kaur, I.P., 2013b. Curcumin loaded solid lipid nanoparticles: an efficient formulation approach for cerebral ischemic reperfusion injury in rats. Eur. J. Pharm. Biopharm. 85, 339-345.

Kim, Y.T., Caldwell, J.M., Bellamkonda, R.V., 2009. Nanoparticle-mediated local delivery of methylprednisolone after spinal cord injury. Biomaterials 30, 2582-2590.

Kim, G.D., Park, Y.S., Jin, Y.H., Park, C.S., 2015. Production and applications of rosmarinic acid and structurally related compounds. Appl. Microbiol. Biotechnol. 99, 2083-2092.

Kumar, P., Choonara, Y.E., Modi, G., Naidoo, D., Pillay, V., 2014. Nanoparticulate strategies for the five R's of traumatic spinal cord injury intervention: restriction, repair regeneration, restoration and reorganization. Nanomedicine 9, 331-348.

Kumar, P., Sharma, G., Kumar, R., Singh, B., Malik, R., Katare, O.P., Raza, K., 2016. Promises of a biocompatible nanocarrier in improved brain delivery of quercetin: biochemical, pharmacokinetic and biodistribution evidences. Int. J. Pharm. 515, 307-314.

Lajtha, A., Perez-Polo, J.R., Rossner, S., 2009. Handbook of Neurochemistry and Molecular Neurobiology - Development and Aging Changes in the Nervous System. Springer, Germany.

Lazar, A.N., Mourtas, S., Youssef, I., Parizot, C., Dauphin, A., Delatour, B., Antimisiaris, S.G., Duyckaerts, C., 2013. Curcumin-conjugated nanoliposomes with high affinity for A $\beta$ deposits: possible applications to Alzheimer disease. Nanomed. Nanotechnol. Biol. Med. 9, 712-721.

Li, F., Gong, Q., Dong, H., Shi, J., 2012. Resveratrol, a neuroprotective supplement for Alzheimer's disease. Curr. Pharm. Des. 18, 27-33.

Lin, F., Zhang, C., Chen, X., Song, E., Sun, S., Chen, M., Pan, T., Deng, X., 2015. Chrysophanol affords neuroprotection against microglial activation and free radicalmediated oxidative damage in BV2 murine microglia. Int. J. Clin. Exp. Med. 8, 3447-3455.

Loureiro, J.A., Andrade, S., Duarte, A., Neves, A.R., Queiroz, J.F., Nunes, C., Sevin, E. Fenart, L., Gosselet, F., Coelho, M.A., Pereira, M.C., 2017. Resveratrol and grape extract-loaded solid lipid nanoparticles for the treatment of Alzheimer's disease. Molecules 22, 277-293.

Lúcio, M., Lima, J.L., Reis, S., 2010. Drug-membrane interactions: significance for medicinal chemistry. Curr. Med. Chem. 17, 1795-1809.

Lun, H., Yu, X., Bendayan, R., 2012. Nanotechnological advances for the delivery of CNS therapeutics. Adv. Drug Del. Rev. 64, 686-700.

Manach, C., 2004. Polyphenols : food sources and bioavailability. Am. J. Clin. Nutr. 79, $727-747$.

Martin-Banderas, L., Holgado, M.A., Venero, J.L., Alvarez-Fuentes, J., Fernandez-Arevalo, M., 2011. Nanostructures for drug delivery to the brain. Curr. Med. Chem. 148, 5303-5321.

McClements, D.J., 2012. Nanoemulsions versus microemulsions: terminology, differences, and similarities. Soft Matter 8, 1719-1729.

Medina, C., Santos-Martinez, M.J., Radomski, A., Corrigan, O.I., Radomski, M.W., 2007. Nanoparticles: pharmacological and toxicological significance. Br. J. Pharmacol. 150, 552-558.

Meng, F., Asghar, S., Gao, S., Su, Z., Song, J., Huo, M., Meng, W., Ping, Q., Xiao, Y., 2015. A novel LDL-mimic nanocarrier for the targeted delivery of curcumin into the brain to treat Alzheimer's disease. Colloids Surf. B. Biointerfaces 134, 88-97.

Modi, G., Pillay, V., Choonara, Y.E., Ndesendo, V.M., du Toit, L.C., Naidoo, D., 2009. Nanotechnological applications for the treatment of neurodegenerative disorders. Prog. Neurobiol. 88, 272-285.

Mourtas, S., Canovi, M., Zona, C., Aurilia, D., Niarakis, A., La Ferla, B., Salmona, M., Nicotra, F., Gobbi, M., Antimisiaris, S.G., 2011. Curcumin-decorated nanoliposomes with very high affinity for amyloid- $\beta 1-42$ peptide. Biomaterials 32 , 1635-1645.

Mourtas, S., Lazar, A.N., Markoutsa, E., Duyckaerts, C., Antimisiaris, S.G., 2014. Multifunctional nanoliposomes with curcumin-lipid derivative and brain targeting functionality with potential applications for Alzheimer disease. Eur. J. Med. Chem. 80, 175-183.

Mu, X., He, G.R., Yuan, X., Li, X.X., Du, G.H., 2011. Baicalein protects the brain against neuron impairments induced by MPTP in C57BL/6 mice. Pharmacol. Biochem. Behav. 98, 286-291.

Mura, S., Nicolas, J., Couvreur, P., 2013. Stimuli-responsive nanocarriers for drug delivery. Nat. Mater. 12, 991-1003.

Narasimhan, R., Vaithiyanathan, M., Janardanam, V., 2011. Neuroprotective effect of sesamol in glioma induced in rats. Biomed. Int. 2, 22-27.

Nasr, M., 2016. Development of an optimized hyaluronic acid-based lipidic nanoemulsion co-encapsulating two polyphenols for nose to brain delivery. Drug Deliv. 23, 1444-1452.

Neves, A.R., Lucio, M., Lima, J.L., Reis, S., 2012. Resveratrol in medicinal chemistry: a critical review of its pharmacokinetics, drug-delivery, and membrane interactions. Curr. Med. Chem. 19, 1663-1681.

Neves, A.R., Lucio, M., Martins, S., Lima, J.L., Reis, S., 2013. Novel resveratrol nanodelivery systems based on lipid nanoparticles to enhance its oral bioavailability. Int. J. Nanomed. 8, 177-187.

Neves, A.R., Queiroz, J.F., Reis, S., 2016. Brain-targeted delivery of resveratrol using solid lipid nanoparticles functionalized with apolipoprotein E. J. Nanobiotechnology 14, $1-27$.

Nunes, S., Madureira, R., Campos, D., Sarmento, B., Gomes, A.M., Pintado, M., Reis, F., 2015. Solid lipid nanoparticles as oral delivery systems of phenolic compounds: overcoming pharmacokinetic limitations for nutraceutical applications. Crit. Rev. Food Sci. Nutr. 57, 1863-1873.

Olbrich, C., Kayser, O., Müller, R.H., 2002. Lipase degradation of Dynasan 114 and 116 solid lipid nanoparticles (SLN)—effect of surfactants, storage time and crystallinity. Int. J. Pharm. 237, 119-128.

Olesen, J., Gustavsson, a., Svensson, M., Wittchen, H.U., Jönsson, B., 2012. The economic cost of brain disorders in Europe. Eur. J. Neurol. 19, 155-162.

Oliveira, A.I., Pinho, C., Sarmento, B., Dias, A.C., 2016. Neuroprotective activity of 
Hypericum perforatum and its major components. Front. Plant Sci. 7, 1004-1019.

Pandita, D., Kumar, S., Poonia, N., Lather, V., 2014. Solid lipid nanoparticles enhance oral bioavailability of resveratrol, a natural polyphenol. Food Res. Int. 62, 1165-1174.

Pardeike, J., Hommoss, A., Müller, R.H., 2009. Lipid nanoparticles (SLN, NLC) in cosmetic and pharmaceutical dermal products. Int. J. Pharm. 366, 170-184.

Pardeshi, C.V., Belgamwar, V.S., 2013. Direct nose to brain drug delivery via integrated nerve pathways bypassing the blood-brain barrier: an excellent platform for brain targeting. Expert Opin. Drug Deliv. 10, 957-972.

Pardridge, W.M., 2003. Blood-brain barrier drug targeting: the future of brain drug development. Mol. Interventions 3, 90-105.

Phachonpai, W., Wattanathorn, J., Muchimapura, S., Tong-Un, T., Preechagoon, D., 2010. Neuroprotective effect of quercetin encapsulated liposomes: a novel therapeutic strategy against Alzheimer's disease. Am. J. Appl. Sci. 7, 480-485.

Pinnen, F., Sozio, P., Cacciatore, I., Cornacchia, C., Mollica, A., Iannitelli, A., D’Aurizio, E., Cataldi, A., Zara, S., Nasuti, C., Di Stefano, A., 2011. Ibuprofen and glutathione conjugate as a potential therapeutic agent for treating Alzheimer's disease. Arch. Pharm. 344, 139-148.

Puglia, C., Frasca, G., Musumeci, T., Rizza, L., Puglisi, G., Bonina, F., Chiechio, S., 2012. Curcumin loaded NLC induces histone hypoacetylation in the CNS after intraperitoneal administration in mice. Eur. J. Pharm. Biopharm. 81, 288-293.

Pujara, N.D., 2012. Self emulsifying drug delivery system: a novel approach. J. Pharm. Res. 5, 500-504.

Qi, X., Wang, L., Zhu, J., Hu, Z., Zhang, J., 2011. Self-double-emulsifying drug delivery system (SDEDDS): a new way for oral delivery of drugs with high solubility and low permeability. Int. J. Pharm. 409, 245-251.

Qi, J., Zhuang, J., Lu, Y., Dong, X., Zhao, W., Wu, W., 2017. In vivo fate of lipid-based nanoparticles. Drug Discov. Today 22, 166-172.

Ramachandran, S., Thangarajan, S., 2016. A novel therapeutic application of solid lipid nanoparticles encapsulated thymoquinone (TQ-SLNs) on 3-nitroproponic acid induced Huntington's disease-like symptoms in wistar rats. Chem. Biol. Interact. 256 25-36.

Ramalingam, P., Ko, Y.T., 2015. Enhanced oral delivery of curcumin from N-trimethyl chitosan surface-modified solid lipid nanoparticles: pharmacokinetic and brain distribution evaluations. Pharm. Res. 32, 389-402.

Ramalingam, P., Ko, Y.T., 2016. Improved oral delivery of resveratrol from N-trimethy chitosan-g-palmitic acid surface-modified solid lipid nanoparticles. Colloids Surf. B. Biointerfaces 139, 52-61.

Ramassamy, C., 2006. Emerging role of polyphenolic compounds in the treatment of neurodegenerative diseases: a review of their intracellular targets. Eur. J. Pharmacol. 545, 51-64.

Ratnam, D.V., Ankola, D.D., Bhardwaj, V., Sahana, D.K., Kumar, M.N.V.R., 2006. Role of antioxidants in prophylaxis and therapy: a pharmaceutical perspective. J. Control. Release 113, 189-207.

Ray, B., Lahiri, D.K., 2009. Neuroinflammation in Alzheimer's disease: different molecular targets and potential therapeutic agents including curcumin. Curr. Opin. Pharm. 9, 434-444.

Richardson, R.L., Kim, E.M., Shephard, R.A., Gardiner, T., Cleary, J., O’Hare, E., 2002. Behavioural and histopathological analyses of ibuprofen treatment on the effect of aggregated $A \beta(1-42)$ injections in the rat. Brain Res. 954, 1-10.

Rivera, F., Costa, G., Abin, A., Urbanavicius, J., Arruti, C., Casanova, G., Dajas, F., 2008. Reduction of ischemic brain damage and increase of glutathione by a liposomal preparation of quercetin in permanent focal ischemia in rats. Neurotox. Res. 13, 105-114.

Sa, F., Guo, B.J., Li, S., Zhang, Z.J., Chan, H.M., Zheng, Y., Lee, S.M.Y., 2015a. Pharmacokinetic study and optimal formulation of new anti-Parkinson natural compound schisantherin A. Parkinsons Dis. 1-7.

Sa, F., Zhang, L.Q., Chong, C.M., Guo, B.J., Li, S., Zhang, Z.J., Zheng, Y., Hoi, P.M., Lee, S.M., 2015b. Discovery of novel anti-parkinsonian effect of schisantherin a in vitro and in vivo. Neurosci. Lett. 593, 7-12.

Sabogal-Guaqueta, A.M., Munoz-Manco, J.I., Ramirez-Pineda, J.R., Lamprea-Rodriguez, M., Osorio, E., Cardona-Gomez, G.P., 2015. The flavonoid quercetin ameliorates Alzheimer's disease pathology and protects cognitive and emotional function in aged triple transgenic Alzheimer's disease model mice. Neuropharmacology 93, 134-145.

Sachdeva, A.K., Misra, S., Pal Kaur, I., Chopra, K., 2015. Neuroprotective potential of sesamol and its loaded solid lipid nanoparticles in ICV-STZ-induced cognitive deficits: behavioral and biochemical evidence. Eur. J. Pharmacol. 747, 132-140.

Sarkar, S., Das, N., 2006. Mannosylated liposomal flavonoid in combating age-related ischemia-reperfusion induced oxidative damage in rat brain. Mech. Ageing Dev. 127, 391-397.

Sciacca, Michele F., Kotler, Samuel A., Brender, Jeffrey R., Chen, J., Lee, D.-K., Ramamoorthy, A., 2012. Two-step mechanism of membrane disruption by A $\beta$ through membrane fragmentation and pore formation. Biophys. J. 103, 702-710.

Sharma, H.S., 2007. Nanoneuroscience: emerging concepts on nanoneurotoxicity and nanoneuroprotection. Nanomedicine 2, 753-758.

Shi, J., Wang, Y., Luo, G., 2012. Ligustrazine phosphate ethosomes for treatment of Alzheimer's disease, in vitro and animal model studies. AAPS PharmSciTech 13, 485-492.

Silva, G.A., 2006. Neuroscience nanotechnology: progress, opportunities and challenges. Nat. Rev. Neurosci. 7, 65-74.

Silva, B.A., Dias, A.C., Ferreres, F., Malva, J.O., Oliveira, C.R., 2004. Neuroprotective effect of $H$. perforatum extracts on $\beta$-amyloid-induced neurotoxicity. Neurotox. Res. 6 , 119-130.

Silva, B.A., Oliveira, P.J., Dias, A.C.P., Malva, J.O., 2007. Mitochondria as targets for neuronal protection against excitotoxicity: a role for phenolic compounds? Cent. Nerv. Syst. Agents Med. Chem. 7, 205-222.

Silva, B.A., Malva, J.O., Dias, A.C.P., 2008a. St. John's Wort (Hypericum perforatum) extracts and isolated phenolic compounds are effective antioxidants in several in vitro models of oxidative stress. Food Chem. 110, 611-619.

Silva, B.A., Oliveira, P.J., Dias, A., Malva, J.O., 2008b. Quercetin, kaempferol and biapigenin from Hypericum perforatum are neuroprotective against excitotoxic insults. Neurotox. Res. 13, 265-279.

Silva, B.A., Oliveira, P.J., Cristóvão, A., Dias, A.C.P., Malva, J.O., 2009. Biapigenin modulates the activity of the adenine nucleotide translocator in isolated rat brain mitochondria. Neurotox. Res. 17, 75-90.

Smith, A., Giunta, B., Bickford, P.C., Fountain, M., Tan, J., Shytle, R.D., 2010. Nanolipidic particles improve the bioavailability and alpha-secretase inducing ability of epigallocatechin-3-gallate (EGCG) for the treatment of Alzheimer's disease. Int. J. Pharm. 389, 207-212.

Sofroniew, M.V., 2009. Molecular dissection of reactive astrogliosis and glial scar formation. Trends Neurosci. 32, 638-647.

Sonkusare, S.K., Kaul, C.L., Ramarao, P., 2005. Dementia of Alzheimer's disease and other neurodegenerative disorders-memantine, a new hope. Pharmacol. Res. 51, 1-17.

Sood, S., Jain, K., Gowthamarajan, K., 2014. Optimization of curcumin nanoemulsion for intranasal delivery using design of experiment and its toxicity assessment. Colloids Surf. B. Biointerfaces 113, 330-337.

Spuch, C., Saida, O., Navarro, C., 2012. Advances in the treatment of neurodegenerative disorders employing nanoparticles. Recent Pat. Drug Deliv. Formulation 6, 2-18.

Tong-un, T., Muchimapura, S., Phachonpai, W., Wattanathorn, J., 2010. Effects of quercetin encapsulated liposomes via nasal administration: a novel cognitive enhancer. Am. J. Appl. Sci. 7, 906-913.

Torchilin, V.P., 2006. Micellar nanocarriers: pharmaceutical perspectives. Pharm. Res. $24,1-16$.

Tosi, G., Musumeci, T., Ruozi, B., Carbone, C., Belletti, D., Pignatello, R., Vandelli, M.A., Puglisi, G., 2016. The "fate" of polymeric and lipid nanoparticles for brain delivery and targeting: strategies and mechanism of blood-brain barrier crossing and trafficking into the central nervous system. J. Drug Deliv. Sci. Technol. 32, 66-76.

Trautmann, S., Rehm, J., Wittchen, H.-U., 2016. The economic costs of mental disorders. EMBO Rep. 17, 1245-1249.

Tsai, M.J., Wu, P.C., Huang, Y.B., Chang, J.S., Lin, C.L., Tsai, Y.H., Fang, J.Y., 2012. Baicalein loaded in tocol nanostructured lipid carriers (tocol NLCs) for enhanced stability and brain targeting. Int. J. Pharm. 423, 461-470.

Tu, X.K., Yang, W.Z., Shi, S.S., Wang, C.H., Chen, C.M., 2009. Neuroprotective effect of baicalin in a rat model of permanent focal cerebral ischemia. Neurochem. Res. 34, $1626-1634$.

Tuppo, E.E., Arias, H.R., 2005. The role of inflammation in Alzheimer's disease. Int. J. Biochem. Mol. Biol. 37, 289-305.

Üner, M., Yener, G., 2007. Importance of solid lipid nanoparticles (SLN) in various administration routes and future perspective. Int. J. Nanomed. 2, 289-300.

Vanaja, K., Wahl, M.A., Bukarica, L., Heinle, H., 2013. Liposomes as carriers of the lipid soluble antioxidant resveratrol: evaluation of amelioration of oxidative stress by additional antioxidant vitamin. Life Sci. 93, 917-923.

Vedagiri, A., Thangarajan, S., 2016. Mitigating effect of chrysin loaded solid lipid nanoparticles against amyloid $\beta 25-35$ induced oxidative stress in rat hippocampal region: an efficient formulation approach for Alzheimer's disease. Neuropeptides 58, $111-125$.

Walker, J.M., Klakotskaia, D., Ajit, D., Weisman, G.A., Wood, W.G., Sun, G.Y., Serfozo, P., Simonyi, A., Schachtman, T.R., 2015. Beneficial effects of dietary EGCG and voluntary exercise on behavior in an Alzheimer's disease mouse model. J. Alzheimer's Dis. 44, 561-572.

Wang, H., Eckel, R.H., 2014. What are lipoproteins doing in the brain? Trends Endocrinol. Metab. TEM 25, 8-14.

Wang, Y., Xu, H., Fu, Q., Ma, R., Xiang, J., 2011. Protective effect of resveratrol derived from Polygonum cuspidatum and its liposomal form on nigral cells in Parkinsonian rats. J. Neurol. Sci. 304, 29-34.

Wang, J., Wang, H., Zhu, R., Liu, Q., Fei, J., Wang, S., 2015. Anti-inflammatory activity of curcumin-loaded solid lipid nanoparticles in IL-1ß transgenic mice subjected to the lipopolysaccharide-induced sepsis. Biomaterials 53, 475-483.

Wei, Y., Guo, J., Zheng, X., Wu, J., Zhou, Y., Yu, Y., Ye, Y., Zhang, L., Zhao, L., 2014 Preparation, pharmacokinetics and biodistribution of baicalin-loaded liposomes. Int. J. Nanomed. 9, 3623-3630.

Xu, J., Wang, H., Ding, K., Zhang, L., Wang, C., Li, T., Wei, W., Lu, X., 2014. Luteolin provides neuroprotection in models of traumatic brain injury via the Nrf2-ARE pathway. Free Radic. Biol. Med. 71, 186-195.

Yan, J., Zheng, M., Zhang, D., 2014. Chrysophanol liposome preconditioning protects against cerebral ischemia-reperfusion injury by inhibiting oxidative stress and apoptosis in mice. Int. J. Pharmacol. 10, 55-68.

Yang, W., Chen, Y.H., Liu, H., Qu, H.D., 2015. Neuroprotective effects of piperine on the 1-methyl-4-phenyl-1,2,3,6-tetrahydropyridine-induced Parkinson's disease mouse model. Int. J. Mol. Med. 36, 1369-1376.

Ye, Y., Xing, H., Li, Y., 2014. Nanoencapsulation of the sasanquasaponin from Camellia oleifera, its photo responsiveness and neuroprotective effects. Int. J. Nanomed. 9, 4475-4484.

Youdim, M.B.H., Buccafusco, J.J., 2005. Multi-functional drugs for various CNS targets in the treatment of neurodegenerative disorders. Trends Pharmacol. Sci. 26, 27-35.

Yusuf, M., Khan, M., Khan, R.A., Ahmed, B., 2013. Preparation, characterization, in vivo and biochemical evaluation of brain targeted piperine solid lipid nanoparticles in an experimentally induced Alzheimer's disease model. J. Drug Target 21, 300-311.

Zeinali, M., Rezaee, S.A., Hosseinzadeh, H., 2017. An overview on immunoregulatory and anti-inflammatory properties of chrysin and flavonoids substances. Biomed. Pharmacother. 92, 998-1009.

Zhang, H.Y., Tang, X.C., 2006. Neuroprotective effects of huperzine A: new therapeutic targets for neurodegenerative disease. Trends Pharmacol. Sci. 27, 619-625. 
Zhang, C., Ma, J., Fan, L., Zou, Y., Dang, X., Wang, K., Song, J., 2015. Neuroprotective effects of safranal in a rat model of traumatic injury to the spinal cord by antiapoptotic, anti-inflammatory and edema-attenuating. Tissue Cell 47, 291-300.

Zhang, X.Z., Qian, S.S., Zhang, Y.J., Wang, R.Q., 2016. Salvia miltiorrhiza: a source for anti-Alzheimer's disease drugs. Pharm. Biol. 54, 18-24.

Zhao, G., Zang, S.Y., Jiang, Z.H., Chen, Y.Y., Ji, X.H., Lu, B.F., Wu, J.H., Qin, G.W., Guo, L.H., 2011. Postischemic administration of liposome-encapsulated luteolin prevents against ischemia-reperfusion injury in a rat middle cerebral artery occlusion model. J. Nutr. Biochem. 22, 929-936.

Zlokovic, B.V., 2008. The blood-brain barrier in health and chronic neurodegenerative disorders. Neuron 57, 178-201.

Zotova, E., Nicoll, J.A., Kalaria, R., Holmes, C., Boche, D., 2010. Inflammation in

Alzheimer's disease: relevance to pathogenesis and therapy. Alzheimers Res. Ther 2, 1-9. 